\title{
Ductile failure modeling
}

Benzerga, Ahmed Amine; Leblond, Jean Baptiste; Needleman, Alan; Tvergaard, Viggo

\section{Published in:}

International Journal of Fracture

Link to article, DOI:

$10.1007 / \mathrm{s} 10704-016-0142-6$

$10.1007 / \mathrm{s} 10704-016-0159-\mathrm{x}$

Publication date:

2016

Document Version

Peer reviewed version

Link back to DTU Orbit

Citation (APA):

Benzerga, A. A., Leblond, J. B., Needleman, A., \& Tvergaard, V. (2016). Ductile failure modeling. International Journal of Fracture, 201(1), 29-80. https://doi.org/10.1007/s10704-016-0142-6, https://doi.org/10.1007/s10704016-0159-x

\section{General rights}

Copyright and moral rights for the publications made accessible in the public portal are retained by the authors and/or other copyright owners and it is a condition of accessing publications that users recognise and abide by the legal requirements associated with these rights.

- Users may download and print one copy of any publication from the public portal for the purpose of private study or research.

- You may not further distribute the material or use it for any profit-making activity or commercial gain

- You may freely distribute the URL identifying the publication in the public portal

If you believe that this document breaches copyright please contact us providing details, and we will remove access to the work immediately and investigate your claim 


\title{
Ductile Failure Modeling
}

\author{
Amine Benzerga · Jean-Baptiste Leblond · Alan Needleman • Viggo \\ Tvergaard
}

Received: date / Accepted: date

\begin{abstract}
The ...
Keywords Ductile fracture Crack growth Finite Elements
\end{abstract}

\section{Introduction - Viggo}

The role played by void nucleation, growth and coalescence in ductile fracture was identified in the 1940s, [1]. However, it was not until the 1960's that the phenomenology of this process was well documented, [2-5]. In structural metals deformed at room temperature, the voids generally nucleate by decohesion of second phase particles or by particle fracture, and grow by plastic deformation of the surrounding matrix. Void coalescence occurs either by necking down of the matrix material between adjacent voids or by localized shearing between well separated voids, as has been described in a number of previous review papers [6-8].

The first micromechanical studies of void growth were carried out for a single void in an infinite elasticplastic solid, either a circular cylindrical void [9] or a spherical void [10]. A numerical study for a material containing a periodic array of circular cylindrical voids [11] allowed for including the effect of the interaction with neighbouring voids, both in the early

Amine Benzerga and Alan Needleman

Department of Materials Science \& Engineering, Texas A\&M University

Jean-Baptiste Leblond

Institut Jean le Rond d'Alembert, Sorbonne Universités, Université Pierre et Marie Curie

Viggo Tvergaard

Department of Mechanical Engineering, The Technical University of Denmark growth stages and in the final stages approaching coalescence. This numerical study considered a representative unit volume, containing a single void, with appropriate boundary conditions to represent the full material. Such unit cell analyses have become an important tool in the analysis of several different aspects of ductile fracture. It is also appreciated that unit cells containing many voids have advantages over those with only one void, as they can account for differences in void size or spacing and also for localized plastic flow due to void clustering or due to instabilities.

The most widely known porous ductile material model is that developed by Gurson based on micromechanical studies [12,13], using averaging techniques similar to those applied in [14]. Some improvements were early added to this model [15-18], resulting in a modified Gurson model (the so-called GTN model), which has been used extensively in the following years to study a number of different problems. In many applications the material does not contain any voids initially, so the representation of gradual void nucleation during the deformation process is important. Porous ductile material models were also developed early on by fitting experiments for powder metallurgical materials [19], and in fact the approximate yield surfaces obtained by these different methods are in rather good agreement for a given void volume fraction.

The Gurson model is limited by a number of assumptions, e.g. the voids are embedded in a standard Mises solid, and the voids are taken to remain spherical independent of the stress state. At low stress triaxiality, i.e. low mean tensile stress relative to the effective Mises stress, voids tend to elongate, and this has strong influence on predictions of ductile failure. Early studies that extended the Gurson model to account for void shape effects are given in [20-22]. Other early work on 
the effect of shape changes was presented in [23]. Regarding the effect of anisotropy the Gurson model was extended in [24] to consider a spherical void embedded in an elastic-plastic matrix that obeys Hill's quadratic yield condition [25]. This model was further extended in $[26,27]$ to also account for non-spherical voids embedded in the same anisotropic solid.

The final failure in void containing materials typically occurs by void coalescence, where the ligament between neighbor voids necks down to zero thickness and leaves the characteristic fibrous fracture surface. An important contribution to the modeling of this mechanism has been given in [28]. However, quite often the final failure is associated with a shear band instability [29, 30], leading to a so-called void-sheet failure, where voids grow to coalescence inside a narrow layer of material [2] and the fracture surface shows that the voids have been smeared out during coalescence. In materials containing two size scales of voids or inclusions from which voids nucleate, it is sometimes observed that plastic flow localization develops between two larger voids and that final failure involves void-sheet failure by the small scale voids between the larger voids $[31,32]$. In a model of this phenomenon the small scale voids have been represented in terms of the Gurson model and localization leading to void-sheet failure between larger voids has been predicted [33].

Recently there has been increasing interest in the behavior of porous materials under low stress triaxiality, such as simple shear, where the standard material models do not predict void growth to coalescence. Full $3 \mathrm{D}$ analyses for shear specimens containing spherical voids have been carried out [34] in order to model experiments on ductile fracture in double notched tube specimens loaded in combined tension and torsion [35]. In a number of plane strain cell model analyses for a material containing a periodic array of circular cylindrical voids it has been found [36-39] that in stress states similar to simple shear the voids are flattened out to micro-cracks, which rotate and elongate until interaction with neighbouring micro-cracks gives coalescence, where stresses pass through a maximum so that failure is predicted. This mechanism has also been found in 3D for initially spherical voids [40]. Thus, under high stress triaxiality the void volume fraction increases until ductile fracture occurs, whereas the void volume fraction disappears under low stress triaxiality, as the voids become micro-cracks. The significant void shape changes at low stress triaxiality are accounted in the models [2023] mentioned above, but to deal with failure in simple shear the models must be extended to describe void closure into micro-cracks and the interaction between these micro-cracks.
The early constitutive models for porous ductile solids did not incorporate an effect of the third stress invariant $J_{3}$, but recently there has been more focus on this through the effect of the Lode parameter. It has been found in fracture tests under loads including shear [41, 42] that the effective plastic strain does not decrease monotonically with increasing stress triaxiality. This has been further investigated in [43], where tensiontorsion fracture experiments are modelled using an extension of the Gurson model [44], which has been made $J_{3}$ dependent by adding an extra damage term that allows for failure prediction even at zero hydrostatic tension. This extension of the Gurson model [44] has been compared with cell model studies for voids in shear fields [45] and it has been found that the model can capture quantitative aspects of softening and localization in shear. In [43] the tension-torsion fractures are modelled by finding the localization strain in a shear stress state with more or less tension superposed, and it is shown that the failure strain does not vary monotonically with the stress triaxiality.

\section{Void nucleation - Amine}

\section{Void growth - Jean-Baptiste}

\subsection{Generalities}

There are two basic methods to derive "homogenized" models for porous plastic materials depicting the second phase - void growth - of ductile fracture:

- The first was initiated by Gurson [12,13], followed by many others $[20-22,24,46,47,26,27,48-51]$. Its principle consisted in combining the theory of limitanalysis (equivalent to plasticity theory in the absence of elasticity and strain hardening) with homogenization of some "elementary cell" in some plastic porous material. The shape of this cell was "adapted" to that of the enclosed void: spherical/cylindrical for a spherical/cylindrical void, spheroidal and confocal with the void if spheroidal, ellipsoidal and again confocal with the void if ellipsoidal. Conditions of homogeneous boundary strain rate, as proposed by Mandel [52] and Hill [53], were used. The matrix was assumed to obey the von Mises (isotropic) yield criterion or the Hill [25] (orthotropic) criterion.

- The second originated from homogenization methods extending the well-known linear Hashin-Shtrikman bounds to nonlinear composites [54-56], and used a technique of "comparison" with some reference linear material. The early model of Ponte-Castaneda and Zaidman [23], in spite of its accuracy for devi- 
atoric loadings, suffered from a notable overestimation of the overall yield limit under hydrostatic loading. This drawback was remedied in the more recent model of Danas and Ponte-Castaneda $[57,58]$ based on Ponte-Castaneda's [59] "second-order homogenization method". Ponte-Castaneda and Zaidman's [23] yield surface was also very recently improved by Agoras and Ponte-Castaneda [60,61] using an "iterative" approach devised by Ponte-Castaneda [62].

Both approaches are presented in the sequel but with major emphasis on the first one, which has been followed by most authors and used more widely for practical applications. As will be seen, a remarkable, though still incomplete degree of convergence between these approaches is apparent in very recent works.

\subsection{Gurson's model}

\subsubsection{Original form}

Gurson's [13] model was derived from approximate homogenization and limit-analysis of a hollow sphere made of some rigid, ideal-plastic material obeying von Mises's criterion and the associated plastic flow rule, and subjected to conditions of homogeneous boundary strain rate (Mandel [52], Hill [53]). The overall criterion thus obtained reads

$\Phi(\boldsymbol{\Sigma}, f)=\frac{\Sigma_{e q}^{2}}{\bar{\sigma}^{2}}+2 f \cosh \left(\frac{3 \Sigma_{m}}{2 \bar{\sigma}}\right)-1-f^{2} \leq 0$

where $\Sigma_{e q}=\left(\frac{3}{2} \boldsymbol{\Sigma}^{\prime}: \boldsymbol{\Sigma}^{\prime}\right)^{1 / 2}\left(\boldsymbol{\Sigma}^{\prime}\right.$ overall stress deviator) and $\Sigma_{m}=\frac{1}{3} \operatorname{tr} \boldsymbol{\Sigma}$ ( $\boldsymbol{\Sigma}$ overall stress tensor) denote the overall von Mises equivalent and mean stresses, respectively, $\bar{\sigma}$ the matrix yield stress in simple tension and $f$ the porosity (void volume fraction). Gurson [13] also showed that as a consequence of homogenization combined with a classical result of limit-analysis, the normality property obeyed at the microscopic scale is preserved at the macroscopic scale; thus the overall flow rule is a direct consequence of the overall criterion, the overall plastic strain $\mathbf{D}^{p}$ being given by

$\mathbf{D}^{p}=\dot{\Lambda} \frac{\partial \Phi}{\partial \boldsymbol{\Sigma}}(\boldsymbol{\Sigma}, f) \quad, \quad \dot{\Lambda}\left\{\begin{array}{l}=0 \text { if } \Phi(\boldsymbol{\Sigma}, f)<0 \\ \geq 0 \text { if } \Phi(\boldsymbol{\Sigma}, f)=0\end{array}\right.$

where $\dot{\Lambda}$ denotes the overall plastic multiplier. In addition, the classical equation resulting from approximate matrix incompressibility (elasticity being disregarded)

$\dot{f}=(1-f) \operatorname{tr} \mathbf{D}^{p}$

shows that the evolution of the internal parameter $f$ is dictated by the flow rule and thus, by what precedes, by the criterion. Therefore specifying this criterion is, quite remarkably, sufficient to completely define the model.

Gurson's [13] model, in its original form defined by equations $(1,2,3)$, possesses the following nice properties, which may serve for an alternative, less rigorous but more intuitive derivation:

- the criterion reduces to that of von Mises in the limit of a zero porosity $f$;

- for a purely deviatoric loading $\left(\Sigma_{m}=0\right)$, it predicts an overall yield stress equal to $(1-f) \bar{\sigma}$, in agreement with the elementary (but rigorous) inequality $\Sigma_{e q} \leq(1-f) \bar{\sigma}$ resulting from Cauchy-Schwartz's inequality;

- for a purely hydrostatic loading $\left(\Sigma_{e q}=0\right)$, it predicts an overall yield stress equal to $-\frac{2}{3} \bar{\sigma} \ln f$, in agreement with the exact result for a hollow sphere resulting from an elementary calculation;

- for a low porosity $f$ and a high triaxiality $T=$ $\Sigma_{m} / \Sigma_{e q}$, combination of equations $(1,2,3)$ essentially yields (up to some multiplicative factor) Rice and Tracey's [10]'s famous exponential void growth law, derived from approximate limit-analysis of a single void embedded in an infinite matrix;

- it formally looks like (without being completely identical to) that for a hollow cylinder subjected to some axisymmetric loading under conditions of generalized plane strain, the exact form of which is also known from Gurson's [13] work.

Gurson's [13] original reasoning, which involved a somewhat dubious expansion in powers of a parameter which was not really small, was very recently reexamined by Leblond and Morin [63] using more rigorous mathematics, and clarified. The main conclusions of this work were twofold:

- Gurson's criterion (1) provides a rigorous "upper bound" for the exact overall yield locus of the hollow sphere envisaged with the boundary conditions considered, and also as a consequence for that of a Hashin assembly of hollow spheres having identical porosities (the same conclusion was also reached by Benzerga and Leblond [8], using a different argument);

- for the overall criterion, Gurson's expansion procedure converges very quickly, and his first-order criterion is almost identical to the final "converged" one; but this is less true for the overall flow rule, Gurson's first-order truncation of the series involving a $25 \%$ maximum error on the porosity rate (further comments on this point are provided below). 


\subsubsection{Extended forms}

The first extension of Gurson's model defined by equations $(1,2,3)$ is due to Gurson [13] himself, and pertains to strain hardening. He assumed hardening to be of isotropic type at the local scale, the yield stress in pure tension of the material being now, instead of a mere constant $\bar{\sigma}$, a given function $\sigma(\epsilon)$ of the von Mises equivalent cumulated strain $\epsilon$. Instead of extending his approximate homogenization of a hollow sphere made of ideal-plastic material to the hardenable case, he adopted a purely heuristic approach which consisted of assuming that his overall yield criterion (1) remained applicable to such a case, the parameter $\bar{\sigma}$ denoting now some "average value" of the local yield stress $\sigma(\epsilon)$. More precisely, he defined $\bar{\sigma}$ as the value of $\sigma$ corresponding to some "average value", $\bar{\epsilon}$, of $\epsilon$, for which he proposed the following evolution equation:

$\bar{\sigma} \frac{d \bar{\epsilon}}{d t}=(1-f) \boldsymbol{\Sigma}: \mathbf{D}^{p} \quad, \quad \bar{\sigma}=\sigma(\bar{\epsilon})$.

The meaning of equation (4) is that the plastic dissipation $(1-f) \boldsymbol{\Sigma}: \mathbf{D}^{p}$ in the real, inhomogeneously strained material is heuristically identified to that, $\bar{\sigma} d \bar{\epsilon} / d t$, in a fictitious, homogeneously strained material with equivalent cumulated strain $\bar{\epsilon}$ and yield stress $\bar{\sigma}=\sigma(\bar{\epsilon})$. One remarkable feature of equation (4) is that it does not only account for the hardening arising from the deviatoric part of the overall plastic strain rate $\mathbf{D}^{p}$, but also, in in an approximate way, for that arising from its hydrostatic part, that is in fact from void growth.

The extended model thus defined however suffers from the fact that the same parameter $\bar{\sigma}$ enters both the "square" and "cosh" terms of the yield criterion, which means that the effect of strain hardening is implicitly assumed to be the same on the overall yield stresses under purely deviatoric and purely hydrostatic loadings. Leblond et al. [64], using an extension of Gurson's [13] approximate homogenization analysis to the hardenable case, have shown that this is only an approximation which may lead to significant errors on the value of porosity rate. They have proposed a variant of Gurson's criterion (1) in which different macroscopic parameters $\Sigma_{1}, \Sigma_{2}$, instead of the single $\bar{\sigma}$, enter the "square" and "cosh" terms; they have evidenced the improvement thus brought to the prediction of the porosity rate through comparison of the model predictions with the results of some micromechanical numerical simulations of a spherical cell, analogous to those of Koplik and Needleman [28] for a cylindrical one.

Extensions of Gurson's [13] original model to matrices exhibiting kinematic hardening have also been proposed by Mear and Hutchinson [65] (see also Tvergaard [66]) and again Leblond et al. [64]. But these extensions are somewhat hampered by the ambiguities and difficulties arising, already at the local scale, in the definition of a "good" kinematic hardening rule in the context of large strain plasticity.

Another extension pertains to the adaptation of Gurson's [13] model to more realistic, non-spherical cell shapes. In order to bring the model predictions to better agreement with the results of some micromechanical simulations, Tvergaard [16] proposed to modify Gurson's criterion (1) into

$\Phi(\boldsymbol{\Sigma}, f)=\frac{\Sigma_{e q}^{2}}{\bar{\sigma}^{2}}+2 q_{1} f \cosh \left(\frac{3 q_{2} \Sigma_{m}}{2 \bar{\sigma}}\right)-1-q_{3} f^{2} \leq 0$

where $q_{1}, q_{2}, q_{3}$ are heuristic parameters. Most authors have adopted values of $q_{2}$ and $q_{3}$ equal to 1 and $q_{1}^{2}$, respectively; Tvergaard's proposed modification then simply amounts to multiplying the porosity $f$ by the heuristic factor $q_{1}$. Values of this parameter of the order of 1.5 have been proposed both by Tvergaard [16] as just mentioned, from comparisons with micromechanical simulations, and Perrin and Leblond [67], from theoretical arguments.

The physical interpretation of the parameter $q_{1}$ is however multi-faceted. For instance:

- Huang's [68] revision of Rice and Tracey's [10] study of void growth in an infinite medium (zero porosity) led to the conclusion that the prefactor in these authors' exponential void growth law was notably underestimated. Gologanu [69] noted that correcting this underestimation within Gurson's [13] model required introducing a $q_{1}$-parameter of the order of 1.6. The role of this parameter is then to correct inaccuracies occurring in the model in the limit of vanishingly small porosities, and has nothing to do with the shape of the elementary cell.

- Leblond and Morin's [63] study has shown that introduction of a $q_{1}$-parameter depending on the triaxiality $T$, and of the order of 1.25 for small $T$, is necessary to correct the inherent error on the porosity rate made by Gurson's [13] model, as a result of his truncation of a series at the first order. In this context the introduction of $q_{1}$ is necessary even for a spherical elementary cell and a finite, nonzero porosity.

Tvergaard's [16] introduction of the " $q_{i}$ "-parameters was completed (i) by Chu and Needleman [15] by introducing an extra term connected to void nucleation in the evolution law (3) of the porosity, see Section 2 above; and (ii) by Tvergaard and Needleman [18] through a heuristic modification of the porosity in the yield criterion (1) and the associated flow rule (2), aimed at phenomenologically accounting for coalescence of voids, 
see Section 4 below. The resulting "GTN" (GursonTvergaard-Needleman) model has been widely used for numerical, finite-element based simulations of ductile rupture of actual, full-size specimens and structures; a few examples will be provided in Section 7 below.

An important modification of Gurson's [13] evolution law (3) of the porosity was recently proposed by Nahshon and Hutchinson [44]. The aim of this modification was to account in a heuristic way for the development of damage evidenced in micromechanical numerical simulations performed by Tvergaard and coworkers [36-40] under conditions of low or vanishing triaxiality $T$. Equation (3) then becomes

$\dot{f}=(1-f) \operatorname{tr} \mathbf{D}^{p}+k_{\omega} f \omega(\boldsymbol{\Sigma}) \frac{\boldsymbol{\Sigma}^{\prime}: \mathbf{D}^{p}}{\bar{\sigma}}$

where $k_{\omega}$ is a heuristic parameter and the function $\omega(\boldsymbol{\Sigma})$ is defined by

$\omega(\boldsymbol{\Sigma})=1-\left(\frac{27 J_{3}}{2 \Sigma_{e q}^{3}}\right)^{2} \quad, \quad J_{3}=\operatorname{det} \boldsymbol{\Sigma}^{\prime}$.

Nahshon and Hutchinson's [44] equation (6) has often been criticized on the grounds that it is in conflict with equation (3), one of the rare rigorous results derived from homogenization. It is true that, as remarked by the authors themselves, adopting it makes it impossible to retain the interpretation of $f$ as the true void volume fraction; $f$ becomes a heuristic damage parameter analogous to those encountered in the "theory of continuum damage mechanics". But it should be borne in mind that in spite of the limitations of equation (7), it represents a convenient, easy-to-implement heuristic way of accounting for the development of ductile damage at low or vanishing triaxiality. The only alternative is to account in a detailed way for the gradual change of shape of the voids under conditions of low triaxiality, and for the resulting softening of the material; models doing such a job are described in Section 3.3 below, and offer a more rigorous way of predicting ductile damage at low or vanishing triaxiality, but at the expense of considerably greater complexity.

Numerous other extensions of Gurson's [13] analysis and model have been proposed; such extensions include incorporation of Eshelby-type velocity fields in the limit-analysis of the hollow sphere (Monchiet et al. [70]), consideration of a matrix obeying Tresca's criterion instead of that of von Mises (Cazacu et al. [71]), etc. These extensions cannot all be cited here.
3.3 Models incorporating void shape effects

\subsubsection{The GLD model for spheroidal voids}

The GLD (Gologanu-Leblond-Devaux) model developed by Gologanu et al. [20-22] extends Gurson's [13] model by introducing void shape effects, in the simplest case of spheroidal (axisymmetric ellipsoidal), prolate or oblate voids. It was developed in three steps. First, Gologanu et al. [20] extended Gurson's limit-analysis of a hollow sphere by considering a prolate spheroidal void enclosed within a confocal spheroidal cell subjected to some axisymmetric loading; the trial velocity fields they used satisfied conditions of homogeneous strain rate on all spheroids confocal with the void and the external boundary. ${ }^{1}$ Second, Gologanu et al. [20] considered oblate voids, using the same type of representative cell and velocity fields. Third, Gologanu et al. [22] refined the limit-analyses for both prolate and oblate voids by considering more velocity fields, belonging to a general class defined by Lee and Mear [73]. They also extended the model to general loadings in a heuristic way.

In the simpler case of an axisymmetric loading $\left(\Sigma_{x x}=\right.$ $\Sigma_{y y} \neq \Sigma_{z z}$, other $\Sigma_{i j}=0, O z$ denoting the axis of rotational symmetry of the void), the GLD criterion reads

$$
\begin{aligned}
\Phi(\boldsymbol{\Sigma}, f, w)= & \frac{C}{\bar{\sigma}^{2}}\left(\Sigma_{z z}-\Sigma_{x x}+\eta \Sigma_{h}\right)^{2} \\
& +2(1+g)(f+g) \cosh \left(\frac{\kappa \Sigma_{h}}{\bar{\sigma}}\right) \\
& -(1+g)^{2}-(f+g)^{2} \leq 0
\end{aligned}
$$

where $w$ denotes the shape parameter of the void (ratio of its axes in the directions $O z$ and $O x$, respectively), $C, \eta, g$ (the "second porosity") and $\kappa$ coefficients depending on the internal parameters $f$ and $w$, and $\Sigma_{h}$ some weighted average of $\Sigma_{x x}$ and $\Sigma_{z z}$ also depending on $f$ and $w$. The expressions and values of the various coefficients are different for prolate and oblate voids:

- For prolate voids $C$ is nearly unity, $\eta$ is small and $g$ is nil; the criterion thus bears a strong resemblance to that for a spherical void, equation (1), and appears as a kind of interpolation between this criterion and that for a cylindrical void, see Gurson [13].

- For oblate voids $C$ may notably differ from unity, and $\eta$ and $g$ are nonzero; therefore the resemblance with Gurson's criterion (1) is less marked. The main novelty with respect to this criterion is the appearance of the coefficient $g$, which plays the role of a kind of additional porosity since it contributes to the reduction of the reversibility domain. For a

1 An alternative limit-analysis based on velocity fields orthogonal to these spheroids was proposed by Garajeu et al. [72]. 
penny-shaped crack (completely flat void) this "second porosity" equals that defined by a spherical void with the same radius. For such a crack, the appearance of such a quantity in the yield criterion is an obvious necessity, since otherwise it would reduce to that of von Mises, $f$ being zero.

The GLD model involves extra internal parameters with respect to that of Gurson, namely the shape parameter $w$ of the voids and the orientation of these voids (that is of their axis of rotational symmetry), for which evolution parameters are needed.

An expression of $\dot{w}$ based on partially analytical, partially numerical limit-analysis, but also on rigorous results for nonlinear composites derived by PonteCastaneda [54], Willis [55] and Michel and Suquet [56] from extensions of Hashin-Shtrikman's theory to the nonlinear case, was proposed by Gologanu et al. [22]. This expression notably accounted for the fact first evidenced by Budiansky et al. [74], and later confirmed by many authors, that a spherical void subjected to some axisymmetric loading with major axial stress $\left(\Sigma_{z z}>\right.$ $\Sigma_{x x}=\Sigma_{y y}$ ) tends to become oblate, instead of prolate, at high triaxialities, in contradiction with what one would intuitively expect and indeed occurs in an elastic material.

With regard to the orientation of the axis of rotational symmetry of the voids, Gologanu et al. [22] simply assumed that the rate of rotation of this axis was equal to the global rate of rotation of the material. This meant neglecting the effect of the global strain rate upon the rotation of the void, and represented an oversimplification which has been justifiably criticized and improved by Scheyvaerts et al. [75].

The GLD model has been used notably to develop coalescence models, for the prediction of the evolution in time of the inter-void distance in the various directions of space; this distance indeed plays an essential role in such models. More details will be provided in Section 4 below.

\subsubsection{The Madou-Leblond model for ellipsoidal voids}

The hypothesis made by Gologanu et al. [20-22] of spheroidal voids raises difficulties in the application of their model, because the three axes of the voids, even if initially equal, almost always take distinct values upon deformation; thus more or less dubious hypotheses must be introduced to replace the real voids by some "equivalent spheroidal voids" having only two distinct axes. This was the motivation for Madou and Leblond's [4851] extension of the GLD model to general ellipsoidal voids having three distinct axes. Again, this extension was done in several steps.
In a first step [48], Madou and Leblond extended Gologanu et al.'s [20,21] approximate limit-analysis of spheroidal cells containing confocal spheroidal voids to ellipsoidal cells containing confocal ellipsoidal voids; to do so, they used a family of velocity fields just discovered by Leblond and Gologanu [76], extending those used by Gologanu et al. [20,21], which satisfied conditions of homogeneous strain rate on an arbitrary family of confocal ellipsoids. In a second step [49], they refined the limit-analysis, first for hydrostatic loadings by performing numerical limit-analyses of the ellipsoidal hollow cells considered, second for deviatoric ones by using Ponte-Castaneda's [54], Willis's [55] and Michel and Suquet's [56] results for nonlinear composites mentioned above. In a third step [50], they performed a large number of finite-element-based limit-analyses of ellipsoidal hollow cells aimed at assessing the quality of the approximate yield criterion proposed. In a final paper [51], they proposed evolution equations for the length and orientation of the axes of the voids, by combining "elastic evolution equations" proposed by Ponte-Castaneda and Zaidman [23] and Kailasam and Ponte-Castaneda [77] with heuristic "plastic corrections" determined numerically.

Madou and Leblond $[48,49]$ showed that with a number of approximations, one may derive, quite remarkably, a criterion of the same basic type as that in the GLD model, equation (8):

$$
\begin{aligned}
\Phi\left(\boldsymbol{\Sigma}, f, w_{1}, w_{2}\right)= & \frac{\mathcal{Q}(\boldsymbol{\Sigma})}{\bar{\sigma}^{2}}+2(1+g)(f+g) \cosh \left[\frac{\mathcal{L}(\boldsymbol{\Sigma})}{\bar{\sigma}}\right] \\
& -(1+g)^{2}-(f+g)^{2} \leq 0
\end{aligned}
$$

where $w_{1}$ and $w_{2}$ are the two shape parameters of the void (ratios of two axes over the third one), $\mathcal{Q}(\boldsymbol{\Sigma})$ a quadratic form of the components of the tensor $\boldsymbol{\Sigma}$ and $\mathcal{L}(\boldsymbol{\Sigma})$ a linear form of its sole diagonal components. The various coefficients involved here depend on $f, w_{1}$ and $w_{2}$; their expressions do not, in the spheroidal case, exactly match those in the GLD model because the approximations used to derive them are slightly different, but the predictions of the two models, in this specific case, are nevertheless very close.

Just like the GLD model, Madou and Leblond's [48-51] model is supplemented with evolution equations for the length and orientation of the axes of the void. Madou and Leblond [51] have proposed to consider, rather than these quantities separately, the quadratic form $\mathcal{P}(\mathbf{u})$ characterizing the geometry of the ellipsoidal void, defined by

$\mathcal{P}(\mathbf{u})=\frac{\left(\mathbf{u . e}_{x}\right)^{2}}{a^{2}}+\frac{\left(\mathbf{u . e}_{y}\right)^{2}}{b^{2}}+\frac{\left(\mathbf{u}_{\mathbf{e}} \mathbf{e}_{z}\right)^{2}}{c^{2}}$ 
where $\mathbf{u}$ denotes an arbitrary vector, $a, b, c$ the principal semi-axes of the void and $\mathbf{e}_{x}, \mathbf{e}_{y}, \mathbf{e}_{z}$ the corresponding unit vectors. (The boundary of the void is defined by the equation $P(\mathbf{O M})=1$ where $O$ denotes its center and $M$ the current point). At each instant, the semi-axes and orientations of the void may be obtained through elementary diagonalization of the $3 \times 3$ symmetric matrix of $P(\mathbf{u})$, so that it suffices to specify the evolution equation of this quadratic form instead of those of $a, b$, $c, \mathbf{e}_{x}, \mathbf{e}_{y}, \mathbf{e}_{z}$ individually; such an evolution equation is much better adapted to the numerical implementation of the model since it is free of singularities when two axes becomes equal. Madou and Leblond [51] showed that the evolution of the matrix $\mathbf{P}$ of the quadratic form $P(\mathbf{u})$ is governed by the equation

$\dot{\mathbf{P}}+\mathbf{P} \cdot\left(\mathbf{D}^{v}+\boldsymbol{\Omega}^{v}\right)+\left(\mathbf{D}^{v}+\boldsymbol{\Omega}^{v}\right)^{T} \cdot \mathbf{P}=\mathbf{0}$,

where $\mathbf{D}^{v}$ and $\boldsymbol{\Omega}^{v}$ denote the strain and rotation rate tensors of the void. This reduced the problem to specifying suitable expressions for $\mathbf{D}^{v}$ and $\boldsymbol{\Omega}^{v}$.

Madou and Leblond [51] then proposed to adopt the following heuristic extensions of Ponte-Castaneda and Zaidman's [23] and Kailasam and Ponte-Castaneda's [77] "elastic expressions" of $\mathbf{D}^{v}$ and $\boldsymbol{\Omega}^{v}$ :

$$
\left\{\begin{array}{l}
\mathbf{D}^{v}=\mathbf{L} . \mathbf{D} \\
\boldsymbol{\Omega}^{v}=\boldsymbol{\Omega}+\mathbf{R} . \mathbf{D}
\end{array}\right.
$$

where $\mathbf{D}$ and $\boldsymbol{\Omega}$ are the global strain and rotation rate tensors and $\mathbf{L}$ and $\mathbf{R}$ 4-th order "plastic localization tensors". The expressions of these tensors were related to those of their elastic counterparts $\mathbf{L}^{e}, \mathbf{R}^{e}$ defined by Ponte-Castaneda and Zaidman [23] and Kailasam and Ponte-Castaneda [77], through multiplication of some of their components by some heuristic correction factors, obtained through a large number of finite element simulations of ellipsoidal hollow cells.

The novelties brought by Madou and Leblond's [4851] model with respect to that of Gurson [13] will now be illustrated by displaying the predictions it makes for the evolution of the overall stress, the porosity, the orientation of the void's axes and the length of these axes, in a few simple, typical cases. These cases have been considered in some micromechanical finite element simulations of Nielsen et al. [40], which are independent of any homogenized model and may therefore be used as references to assess the quality of the model predictions. These simulations consider initially parallelepipedic elementary cells containing an initially spherical void (Fig. 1). A zero orthogonal displacement is imposed on the faces $X_{3}= \pm C_{0}$ (plane strain condition), whereas rigorous periodic conditions are enforced on the faces $X_{1}= \pm A_{0}$, and uniform displacements $U_{1}$ and $U_{2}$ are imposed on the face $X_{2}=B_{0}$, the face $X_{2}=-B_{0}$ being clamped (these conditions are almost equivalent to periodic ones if $B_{0}$ is large enough). The ratio $U_{2} / U_{1}$ is adjusted at every step of the calculation so as to ensure a constant value of the ratio $\kappa=\Sigma_{22} / \Sigma_{12}$ characterizing the importance of the overall shear stress. Possible contact between the void faces is accounted for through some classical penalty method. The material obeys a von Mises criterion with isotropic hardening; the material data are provided in Nielsen et al. [40].

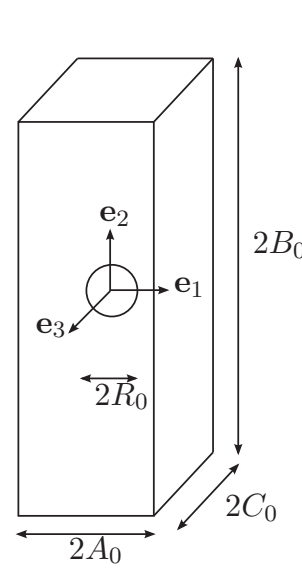

(a) Initial configuration

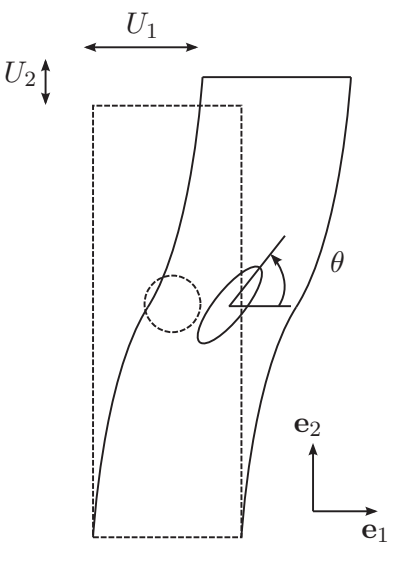

(b) Deformed configuration
Fig. 1: Elementary cell considered by Nielsen et al. [40]

The predictions of Madou and Leblond's [48-51] model are obtained by considering the entire cell as a single element obeying this model, and calculating the response of this element using the finite element programme SYSTUS developed by ESI-Group. Figure 2, borrowed from Morin's thesis [78], compares the predictions of Madou and Leblond's model (ML) with the numerical results of Nielsen et al. [40] (Num), for the normalized overall shear stress $\Sigma_{12} / \sigma_{0}\left(\sigma_{0}=\right.$ initial yield stress $)$ and the normalized porosity $f / f_{0}\left(f_{0}=\right.$ initial porosity). These quantities are plotted versus the "shear angle" defined as $\Psi \equiv \arctan \left[U_{1} /\left(2 B_{0}+U_{2}\right)\right]$. The agreement between numerical and theoretical results is especially good for the shear stress (Fig. 1a). This agreement is no great wonder before the sharp decrease of the stress due to coalescence because the influence of damage is small then; but it is much more significant at and after the onset of this decrease, since it means that the model is able to correctly reproduce coalescence induced by damage due to void shape changes under conditions of low triaxiality, which Gurson's [13] model would completely fail to do. (Coalescence is accounted for using Tvergaard and Needleman's [18] heuristic suggestion, which introduces two 
adjustable parameters; but doing so in the context of Gurson's model would not suffice to induce a significant stress drop, in the absence of significant void growth). For the normalized porosity (Fig. 1b), the reproduction of the numerical results by the model is also quite good. Again, the predictions of Gurson's [13] model would not be so good; for $\kappa=0.25$ for instance, it would predict an increase of the porosity because of the slightly positive triaxiality, in contrast to the numerical results.

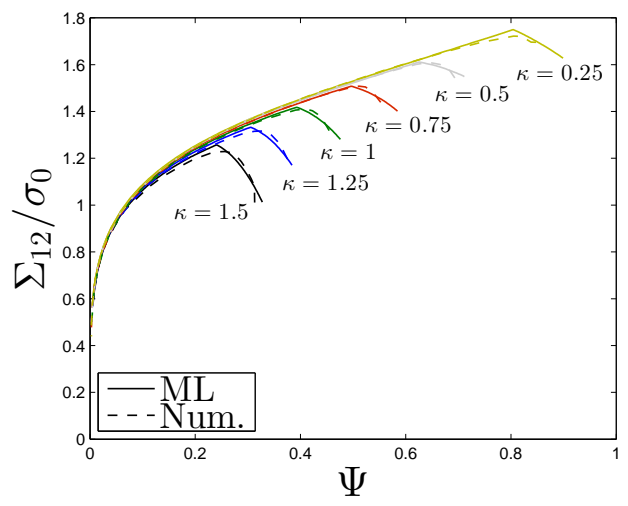

(a) Shear stress

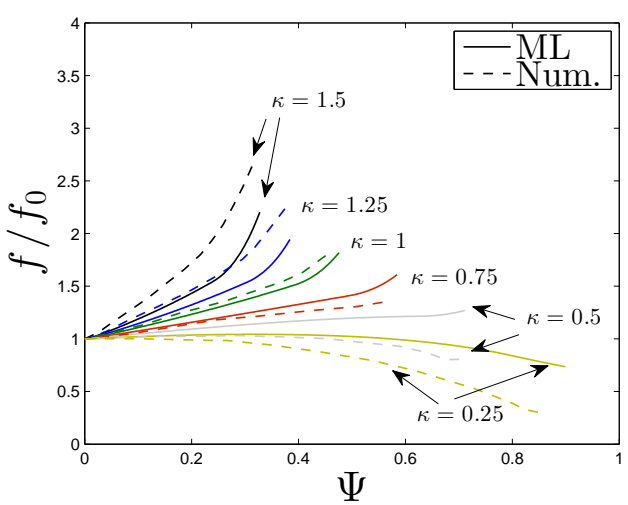

(b) Normalized porosity

Fig. 2: Comparison of the predictions of Madou and Leblond's [48-51] model with the numerical results of Nielsen et al. [40]

Figure 3, again borrowed from Morin's thesis [78], compares numerical results and model predictions for the void orientation characterized by the angle $\theta$ defined in Fig. 1b, and the normalized axes $R_{i} / R_{0}\left(R_{0}=\right.$ radius of the initially spherical void). All features are acceptably reproduced by the model. ${ }^{2}$ Also, the markedly dif-

2 There is a slight gap between numerical and theoretical results for the orientation angle $\theta$; but this gap is present from the very start of the loading (and not compensated afterwards), which indicates that it may be due to the difficulty, ferent evolutions of the void's axes illustrate the impossibility, in such a case, of using the GLD model based on the assumption of equality of two axes.

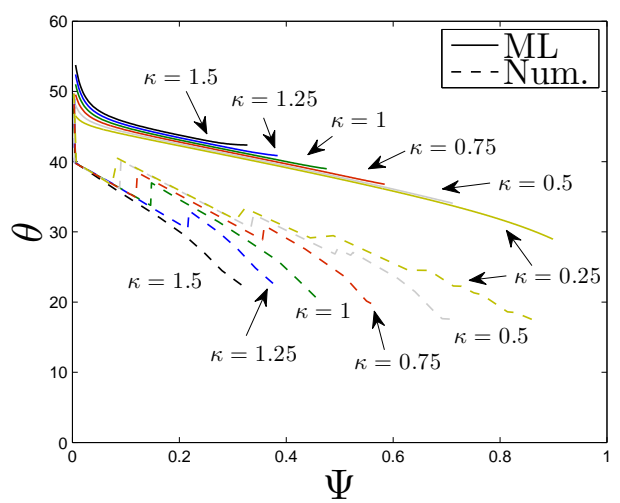

(a) Void orientation

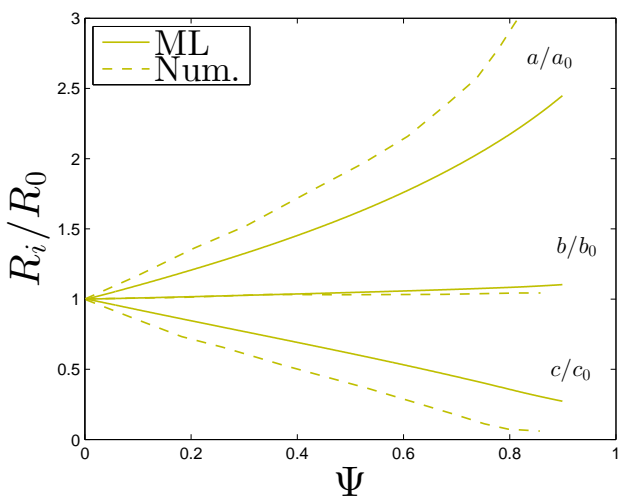

(b) Normalized void axes $(\kappa=0.25)$

Fig. 3: Comparison of the predictions of Madou and Leblond's [48-51] model with the numerical results of Nielsen et al. [40]

A final remark on the approach of ductile fracture based on limit-analysis of elementary hollow cells initiated by Gurson [12,13], resulting from what precedes, is that it has in time borrowed more and more results from the parallel approach developed by Ponte-Castaneda and coworkers, based on the pioneering works of PonteCastaneda [54], Willis [55] and Michel and Suquet [56]. Indeed Gurson's [13] work had no connection whatsoever with this (then inexistent) parallel approach, but Gologanu et al. [22] employed it to refine their evolution equation for the shape parameter of a spheroidal void, and Madou et al. [49,51] used it twice, first to refine their criterion in the case of predominantly deviatoric

when examining the numerical results, of defining an orientation angle for an almost spherical, but not strictly ellipsoidal cavity. 
loadings, second as a basis for the development of evolution equations for the length and orientation of the void axes.

\subsubsection{The models of Ponte-Castaneda and coworkers}

As mentioned above, the models developed by PonteCastaneda and coworkers find their origin in extensions of Hashin-Shtrikman's well-known homogenization theory of random elastic composites to nonlinear behaviors, developed independently in the early 1990's by Ponte-Castaneda [54], Willis [55] and Michel and Suquet [56]. They all rely on a procedure of "comparison" of the real, nonlinear composite with some "reference" linear composite, the number of phases and properties of which may be chosen at will (with some constraints) and optimized, the difficulty being of course that the more complex the reference composite, the harder the resulting calculations. The models proposed have been developed in a number of papers, the most important of which (in the authors' opinion) are mentioned below:

- Ponte-Castaneda and Zaidman [23]'s model first made a relatively simple use of Ponte-Castaneda's [54], Willis's [55] and Michel and Suquet's [56] results, to extend a rigorous elastic bound of Willis [79] for materials having an overall "ellipsoidal symmetry" (extension of the classical spherical symmetry) to nonlinear, plastic or viscoplastic materials. The model was completed by Kailasam and Ponte-Castaneda [77] by defining evolution equations for the length and orientation of the void axes.

The predictions of this early model were quite accurate for low triaxialities, but for high ones the stresses were considerably overestimated. One possible qualitative explanation of these phenomena lies in the range of variation of the local strains, and therefore of the "secant moduli" (ratios of the local stresses over the local strains), depending on the overall stress state. Indeed it is probable that the comparison with some reference linear material is quite relevant if the spatial fluctuations of the secant moduli are moderate, but less so when they become large. Now the fluctuations of the local strains dictating those of the secant moduli are moderate for essentially deviatoric loadings, but not so for essentially hydrostatic ones because the expansion of the voids generates high strains in their vicinity. This suggests that the comparison with some reference linear material must become less and less pertinent when the triaxiality increases.

- In order to remedy this deficiency of the linear comparison procedure, Ponte-Castaneda [59] devised a "second-order" method which was applied by Danas and Ponte-Castaneda $[57,58]$ to the case of porous, plastic or viscoplastic materials. The definition of the new model involved an ad hoc scheme of interpolation and extrapolation aimed at enforcing the exact coincidence of its predictions with those of Gurson's model in the case of spherical/cylindrical voids subjected to hydrostatic overall stress states. This meant dropping to some extent the linear comparison procedure and borrowing instead elements from the alternative Gurson-type approach. The result was a considerable improvement of the model predictions for high triaxialities with respect to PonteCastaneda and Zaidman [23]'s model. The rigorous "bounding properties" of the model were however lost in the process.

- Very recently, Agoras and Ponte-Castaneda [60,61] used an "iterative procedure" just devised by PonteCastaneda [62] to generate another model free of the deficiencies of that of Ponte-Castaneda and Zaidman [23] at high triaxialities. The predictions of this model for purely hydrostatic loadings exactly coincided with the exact results known for a Hashin assembly of homothetical hollow spheres on the one hand, and for the so-called "infinite-rank sequentially laminated microstructures" defined and studied by Idiart $[80,81]$ on the other hand. The remarkable point in this new improved model was that it was obtained without any ad hoc adjustment. However it was not clear whether the model did or did not possess rigorous bounding properties applicable in general; these bounding properties were established only for the two special microstructures just mentioned, the second of which is of little practical relevance since the hypothesis of an infinite sequence of separations of scales it makes can obviously never be met.

It may be noted that Danas and Ponte-Castaneda's $[57,58]$ borrowing of elements of the approach based on limit-analysis of elementary cells symetrically reproduces what Madou and Leblond [49,51] did when defining their own model, see Section 3.3.2 above. A certain degree of convergence of the two approaches in recent years is therefore evident. Whether this convergence will ever be complete and the two approaches merge into a single one remains uncertain at present.

\subsection{Models incorporating plastic anisotropy}

Plastic anisotropy has most often, up to now, been accounted for by assuming the sound material to obey Hill's [25] orthotropic criterion. (A few works dealing 
with porous crystal plasticity will not be commented here).

\subsubsection{Benzerga and Besson's model for spherical voids}

Benzerga and Besson [24] were apparently the first to consider spherical voids embedded in a Hill matrix. Their approach was based on an extension of Gurson's [13] limit-analysis of a hollow sphere made of von Mises material to the case of a Hill material. The criterion they obtained reads

$\Phi(\boldsymbol{\Sigma}, f)=\frac{\Sigma_{e q}^{2}}{\bar{\sigma}^{2}}+2 f \cosh \left(\frac{\kappa \Sigma_{m}}{\bar{\sigma}}\right)-1-f^{2} \leq 0$

where $\kappa$ is a coefficient depending on those defining Hill's local criterion.

There are two differences here with respect to Gurson's criterion (1) applicable to a von Mises matrix. First, the "equivalent stress" $\Sigma_{e q}$ in equation (13) must be understood in the sense of Hill instead of that of von Mises (as is obviously required for the criterion to reduce to that of Hill for a zero porosity $f$ ). Second, the coefficient $\kappa$ is not necessarily equal to $3 / 2$.

It must be noted however that the stress $\Sigma_{m}$ involved in the "cosh", which governs void growth, is exactly the same as in Gurson's criterion (1). This notably implies, via the normality property of the plastic flow rule, that the growth of the voids under hydrostatic loading is predicted to be identical in all directions of space, which means that the preferential growth in certain directions induced by plastic anisotropy is neglected. This arises from the fact that the trial velocity fields used by Benzerga and Besson [24] were exactly identical to those of Gurson [13] for a von Mises matrix, and thus disregarded any influence of anisotropy upon the solution velocity field. It is not immediately clear why such a procedure should be reasonable.

In spite of this, several numerical assessments of Benzerga and Besson's [24] criterion (13) (see notably [82]) have evidenced its accuracy, even for purely hydrostatic overall stress states. The probable explanation of this seemingly puzzling success lies in the variational characterization of the overall plastic dissipation and yield locus. Since the overall dissipation is the minimum of the average value of the corresponding local quantity over the space of incompressible and kinematically admissible velocity fields, it is stationary in the vicinity of the solution field. This means that rather large variations of the trial field around the solution field must result in much smaller variations of the overall plastic dissipation and the resulting approximate yield locus, and are therefore tolerable. This remark has played an important role in the development of more refined models summarized below.

\subsubsection{Models for spheroidal voids}

Similar extensions of the GLD model for spheroidal voids in von Mises matrices to Hill matrices were proposed by Monchiet et al. [46,47] using Gologanu et al.'s $[20,20]$ velocity fields adapted to this geometry, and Keralavarma and Benzerga [26,27] using Gologanu et al.'s [22] richer fields. The criteria they obtained were formally analogous to Madou and Leblond's [48-51] criterion (9) for ellipsoidal voids embedded in a von Mises matrix, albeit with different coefficients. Again, numerical assessment of both Monchiet et al.'s [46,47] and Keralavarma and Benzerga's [26,27] criteria evidenced the quality of their predictions in spite, again, of the use of Gologanu et al.'s [20,20,22] basically "isotropic" velocity fields.

\subsubsection{The Morin-Leblond-Kondo model for ellipsoidal voids}

The model developed very recently by Morin et al. [83] seems to represent an ultimate point in the approach of ductile fracture initiated by Gurson, in that it considers general ellipsoidal voids embedded in some anisotropic Hill matrix, without even assuming coincidence of the principal axes of the voids and those of the material. Using the remark made above about the good results obtained by using "isotropic" velocity fields even for anisotropic matrices, not only for spherical voids (see Section 3.4.1) but also spheroidal ones (see Section 3.4.2), Morin et al. [83] applied Leblond and Gologanu's [76] "isotropic" velocity fields, adapted to the general ellipsoidal case, to a Hill material, thus extending Madou and Leblond's [48-51] work for a von Mises material. The criterion they got was formally analogous to that of Madou and Leblond, equation (9), with different coefficients.

In order to get explicit expressions of all of these for all possible values of the parameters of Hill's criterion, it revealed necessary to introduce an assumption of small anisotropy and perform a first-order expansion in the deviation of these parameters from their "isotropic" values. In spite of this approximation, Morin's [78] very recent numerical assessment of Morin et al.'s [83] criterion seems to evidence the accuracy of its predictions, even for moderate, not-so-small anisotropies.

\subsection{Second gradient extension of Gurson's model}

One common feature of all models mentioned above is that, because they incorporate the softening arising from void growth, they predict a potentially unlimited 
localization of damage and strain, which generates various problems of both mathematical nature (occurrence of bifurcations with an infinite number of bifurcated branches, making their choice impossible) and numerical nature (mesh sensitivity in finite element calculations). The problem is general in softening models. It invariably results from the fact that such models are basically of "homogenized" nature, so that they cease to be applicable at the smaller and smaller scales over which the strain ultimately concentrates. ${ }^{3}$

Although other solutions are possible (see for instance Leblond et al.'s [84] proposal to heuristically consider the porosity as a nonlocal variable, the rate of which is given by some convolution integral), a satisfying, although admittedly complex, solution to this problem was proposed some years ago by Gologanu et al. [22]. Instead of adopting a purely phenomenological approach, Gologanu et al. [22] proposed to extend Gurson's [13] limit-analysis of a hollow sphere made of von Mises material and subjected to Mandel's [52] and Hill's [53] conditions of homogeneous boundary strain rate, to conditions of inhomogeneous boundary strain rate; this meant replacing the linear dependence of the velocity on the elementary cell's boundary upon coordinates, by a quadratic dependence. Although such a dependence is still approximate ${ }^{4}$, its aim is to account for possible quick variations of the macroscopic stress and strain rate fields over distances comparable to the size of the cell (that is to the void spacing), precluded by the more usual linear dependence.

In a first step, Gologanu et al. [22] laid foundations of the model by evaluating the overall virtual powers of forces resulting from the boundary conditions adopted. The main result was that the following expression of the density $\mathcal{P}_{i}^{*}$ of the overall virtual power of internal forces:

$\mathcal{P}_{i}^{*}=-\int_{\Omega}\left(\boldsymbol{\Sigma}: \mathbf{D}^{*}+\mathbf{M} \vdots \nabla \mathbf{D}^{*}\right) d \Omega$.

In this expression $\Omega$ denotes the body considered; $\boldsymbol{\Sigma}$ and $\mathbf{D}^{*}$ are the overall stress and virtual strain rate tensors (average values of the corresponding local quantities $\boldsymbol{\sigma}$ and $\mathbf{d}^{*}$ over the representative cell); $\mathbf{M}$ is the third-rank overall moment tensor, defined as the firstorder moment of the tensor $\boldsymbol{\sigma}$ (average value of the tensor $\boldsymbol{\sigma} \otimes \mathbf{x}$ ) over the cell; and $\nabla \mathbf{D}^{*}$ is the macroscopic

\footnotetext{
3 That softening models have their roots in homogenization is very often only implicit, but in the case of Gurson-type models quite clear and explicit.

4 This means that the homogenization procedure based on such boundary conditions intrinsically remains a model, in contrast with other procedures using rigorous, for instance periodic, boundary conditions.
}

gradient of the tensor $\mathbf{D}^{*}$. The equilibrium equations corresponding to this expression of $\mathcal{P}_{i}^{*}$ read

$\Sigma_{i j, j}-M_{i j k, j k}=0 \quad$ in $\Omega$.

Equations (14) and (15) make it clear that what is obtained here is a second-gradient theory. Note that since the local material behavior is described by the standard first-gradient theory of von Mises, this is a result of the sole homogenization process, and more specifically of the assumption of a velocity varying quadratically, instead of linearly, with coordinates on the cell's boundary.

In a second step, Gologanu et al. [22] extended Gurson's [13] limit-analysis of a hollow sphere by considering extra velocity fields statisfying the extended boundary conditions considered. The output was an overall criterion of the form

$$
\begin{aligned}
\Phi(\boldsymbol{\Sigma}, \mathbf{M}, f)= & \frac{1}{\bar{\sigma}^{2}}\left(\Sigma_{e q}^{2}+\frac{Q^{2}}{b^{2}}\right) \\
& +2 f \cosh \left(\frac{3 \Sigma_{m}}{2 \bar{\sigma}}\right)-1-f^{2} \leq 0
\end{aligned}
$$

where $Q^{2}$ denotes some quadratic form of the components of the moment tensor $\mathbf{M}$, and $b$ the radius of the sphere; this quantity physically represents the mean half-distance between neighboring voids and plays the role of some "microstructural distance" in the model.

Equations (15) and (16) permit to qualitatively understand why the appearance of the moment tensor $\mathbf{M}$ in the model leads to some limitation of the localization of strain and damage. Indeed the equilibrium equations (15) imply that $\nabla \boldsymbol{\Sigma}$ behaves like $\nabla \nabla \mathbf{M}$, so that $\boldsymbol{\Sigma}$ behaves like $\nabla \mathbf{M}$. Now assume that the damage tends to indefinitely localize in time. Then $\mathbf{M}$ varies from $\mathbf{0}$ (in the completely damaged zone) to some nonzero value (in the sound zone) over a distance which gradually goes to zero; it follows that its gradient $\nabla \mathbf{M}$, and therefore the stress tensor $\boldsymbol{\Sigma}$ by what precedes, go to infinity. But this is impossible since the criterion (16) limits the components of the latter tensor. Hence the damage cannot indefinitely localize; of course in practice the size of the damaged zone will be comparable to the microstructural distance $b$.

The development of any second-gradient model, if it is to be of any practical use, must necessarily be accompanied by a discussion of its implementation into some finite element code; indeed such an implementation is indispensable for the study of virtually all practically significant problems, and much less straightforward than that of first-gradient models. The main difficulty lies in the necessary evaluation of spatial derivatives of the strains, that is of the second derivatives of the displacements. 
Enakoutsa and Leblond [85] implemented Gologanu et al.'s second-gradient model, following their suggestion, by (i) introducing new nodal degrees of freedom (DOF) aimed at representing the strains; (ii) calculating the derivatives of the strains by using these new DOF in conjunction with the first derivatives of the shape functions; and (iii) enforcing the approximate coincidence of the new DOF and the strains through some penalty method. The drawbacks of such an approach were an awkwardly large number of DOF per node, especially in 3D, and the difficulty of choosing a "good" value for the penalty coefficient, which had to be sufficiently large to be effective, but not too much in order not to generate an ill-conditioned tangent matrix.

In view of these difficulties, Bergheau et al. [86] proposed, in order to calculate the derivatives of the strains, to retain the introduction of new nodal DOF representing strains, but discard Enakoutsa and Leblond's [85] penalty method, and instead write the equality of these DOF and the strains in a weak sense. The left-hand side of the vectorial relation connecting the new DOF to the nodal displacements then involves a "mass matrix" analogous to that encountered in dynamic problems, which Bergheau et al. [86] proposed to lump and invert straightforwardly, so as to express the new DOF explicitly in terms of the displacements and finally eliminate them. In this way the unknowns are reduced to the sole displacements, like for a standard first-gradient model, and the risk of an ill-conditioned tangent matrix is eliminated. ${ }^{5}$

An additional advantage of Bergheau et al.'s [86] implementation of Gologanu et al.'s [22] model is that unlike most implementations of second-gradient models and especially that of Enakoutsa and Leblond [85], it permits to easily mix elements obeying first- and second-gradient models, since the nodal DOF of the two types of models are identical. An example of such a mix will be provided in Section 7 below. Its practical interest is to reduce the length and cost of calculations by using the second-gradient model only in those zones of the structure where it is really needed, that is where damage develops significantly.

\footnotetext{
5 The price to pay is, unfortunately, a larger bandwidth of the stiffness matrix than for a first-gradient model, because this matrix does not only "connect" first-neighbor nodes (contained in the same element), but also "second-neighbor" ones (having a common first neighbor).
}

\section{Void coalescence - Amine}

\section{Localization - Alan \\ 6 Crack growth - Alan}

\section{Applications}

\subsection{GTN analyses for welded joints - Viggo}

In a number of different analyses the ductile fracture models have been applied to study the failure evolution in welded joints. The examples to be considered here will focus on fusion welded specimens, on friction stir welding and on resistance welding, in particular spotwelded specimens.

\subsubsection{Charpy testing of fusion welded specimens}

The Charpy V-notch test is a standard procedure for characterizing the ductile-to-brittle transition in steels, as has been discussed in $[87,88]$. The use of a micromechanically based material model to analyze the failure mode transition in the Charpy test has been proposed in $[89,90]$. In these studies the GTN model has been used to represent ductile fracture by void growth to coalescence, and this is combined with a model for cleavage failure in grains, which represents the brittle mode of failure. Several investigations of cleavage fracture in body-centered-cubic metals have shown that a constant critical stress is a realistic criterion for slip induced cleavage failure in the low temperature range, and it has been assumed in $[89,90]$ that such a constant critical value $\sigma_{c}$ is a sufficiently good criterion in the whole range of temperatures. Then the temperature dependence of the failure mode results from the fact that at lower temperatures the initial yield stress is higher, so that it is more likely to reach the critical stress $\sigma_{c}$ for cleavage before the amount of plastic yielding is sufficient to develop ductile fracture. The effect of a high loading rate on fracture results from the material strainrate sensitivity, accounted for by a visco-plastic GTN model, which makes it more likely to reach the critical stress $\sigma_{c}$ before void coalescence when the Charpy specimen is subjected to an impact load.

This combination of material models has been applied to Charpy specimens for welded joints, first as a plane strain analysis [91] and subsequently as full 3D analyses [92]. In fusion welding new weld material is melted into the joint and on each side of the weld the base material is separated from the weld material by a narrow heat affected zone (HAZ). Usually the material properties are different in these three material zones along the fusion line. In particular the HAZ material 
tends to have a higher initial yield stress, which makes it more likely that the cleavage stress $\sigma_{c}$ will be reached before ductile fracture, and this more brittle response of the HAZ material is a characteristic feature of the mechanical properties of welds.

To test the weld, Charpy specimens are cut out so that they are perpendicular to the weld and parallel to the surface of the welded piece. The specimen can be cut at various depths below the surface of the welded piece, and the notched face of the Charpy specimen is chosen to be either parallel to the surface of the welded piece or perpendicular to this surface. In the first type of specimen Fig. A illustrates how the $\mathrm{V}$-notch is offset by the distance $x_{c}^{2}$ from the centre of the weld to test the notch-sensitivity of each of the three materials in the weld zone. This geometry can be reasonably well approximated by a plane strain analysis [91] and is also analysed in full 3D [92]. Both the 2D and 3D analyses show that the most critical situation, i.e. the lowest work of fracture corresponding the most brittle response, occurs when the specimen is cut such that the region slightly below the notch falls inside the HAZ.

Figs. $\mathrm{Bb}$ and $\mathrm{Bc}$ show two situations where the notched face of the Charpy specimen is perpendicular to the surface of the welded piece. Clearly these situations are fully three dimensional and cannot be approximated by a planar analysis. Wherever the notch is located inside weld zone some of the material slightly below the notch is inside the HAZ, but still the absorbed energy varies with the location of the notch, as illustrated in Fig. C. The stress state is more constrained in the centre of the specimen than near the free sides, so the stresses tend to be higher near the centre, where brittle fracture might initiate in the HAZ below the notch.

Fig. A. (a) In-plane geometry of the Charpy specimen. (b) Geometry of the fusion-weld. (From [92]). This is Fig. 1 in [92]

Fig. B. Examples of weld configurations analyzed, (1) is the base material, (2) is the weld material, (3) is HAZ. (a) $x_{c}^{2}=6.5 \mathrm{~mm}$, (b) $x_{c}^{2}=4 \mathrm{~mm}$ (rotated 90 deg.), (c) $x_{c}^{2}=7 \mathrm{~mm}$ (rotated $90 \mathrm{deg}$.).(From [92]). This is Fig. 2 in [92]

Fig. C. Work to fracture vs. the distance $x_{c}^{2}$ from the centre of the weld to the notch (rotated 90 deg.). (From [92]). This is Fig. 12 in [92]

\subsubsection{Failure in friction stir welded joints}

Friction stir welding is a relatively new solid state process for joining a variety of different materials. The basis of the process is a spinning tool consisting of a pin and a shoulder plate which is lowered into the weldline between two metal sheets until the shoulders are pressed in contact with the sheets to be welded. When the spinning tool is moved forward along the weldline a joint is created due to friction heating and extensive deformation of the material in the stir zone. The heat and deformation created between the shoulder plate and the material to be welded, results in a difference in the microstructure and thereby the mechanical properties between the top and bottom of the weld.

Ductile fracture in a friction stir welded aluminium plate has been analyzed in [93,94]. This has been done by studying tensile test specimens cut out of the plate perpendicular to the weld, and it is found that the damage development and the position of the final fracture are strongly affected by variations in the yield stress profile transverse to the weldline. Fig. 4 shows typical variations of the yield stress near the weld, normalized by the yield stress of the base material. The three curves shown in Fig. 4(b) give the yield stress variation at the bottom of the weld $\left(x^{2}=0\right)$ and at the middle and the top. As is seen in Fig. 4 the central part of the weld is called the nugget zone (NG), while the edge parts with lowest yield stress are called the thermo-mechanically affected zones (TMAZ). The rotating weld tool moving along the weldline will tend to give a difference between the advancing and the retreating sides of the weld, but in $[93,94]$ the resulting slight non-symmetry of the yield stress profiles has been neglected, thus assuming the symmetric distributions shown in Fig. 4. Also the power hardening exponent is assumed to vary with the yield stress, by using an empirical relationship. The volume fraction of void nucleating particles is assumed to be the same throughout the welded plate.

The analyses in [93] are based on the viscoplastic version of the GTN model, assuming that voids nucleate according to a plastic strain controlled rule, and that fracture occurs when the voids have grown to coalescence. In [94] the same constitutive model is used for some computations, but here the predictions are compared with results of the modified material model proposed in [44]. In this model an extra damage term has been introduced to be able to predict failure at low stress triaxiality. This modification is purely phenomenological, so that the damage parameter $f$ may not be considered as a void volume fraction when [44] has been applied. The amplitude of the extra damage term is denoted $k_{\omega}$, if this amplitude is zero the GTN model is applied, while if $k_{\omega}>0$ the modified material model is applied.

Fig. 5 shows load vs. axial strain curves both for plane strain calculations and for full 3D calculations, in cases where $\sigma_{y}^{(T M A Z)} / \sigma_{y}^{(b)}=0.8$ and $\sigma_{y}^{(N G)} / \sigma_{y}^{(b)}=1.0$. In the $2 \mathrm{D}$ analyses ductile failure is predicted noticeably later by the GTN model than by the modified model, 
because here a rather dominant localized shearing develops in the TMAZ region where the initial yield stress is lower. The difference is smaller in the $3 \mathrm{D}$ analyses.

Contours of the damage $f$ are shown in Fig. 6 for the material properties also considered in Fig. 5 (with $k_{\omega}=3$ ), but for a wider 3D specimen than those considered in Fig. 5 (the figure shows the full plate thickness but only half of the width, due to symmetry). At the first stage the localized shearing in the TMAZ region is clearly visible by the concentrated damage, while in the later stage a broad through-thickness shear region has developed from the centre of the weld part of the specimen.

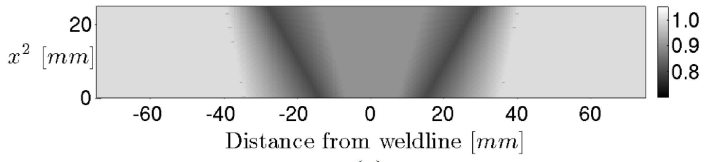

(a)

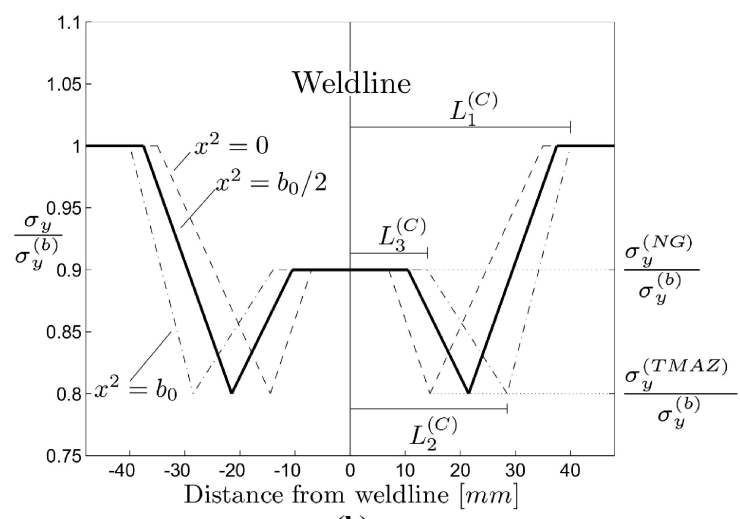

(b)

Fig. 4: Characteristic yield stress variation transverse to the weldline for friction stir welded aluminium alloy; (a) yield stress in weld cross-section; (b) modelled yield stress profile at the top, bottom and middle of the weld cross-section (from [94])

\subsubsection{Resistance spot welded specimens}

Resistance spot welding is a well known method for joining a variety of thin sheet metals. One of the methods used for testing the strength of spot welds is a tensile test for a shear-lab specimen, where two plate strips are welded together by a single spot weld. The failure of such a shear-lab test made of DP600-steel has been analyzed in [95], using the GTN model. Vickers hardness measurements on the cross-section of welds have been

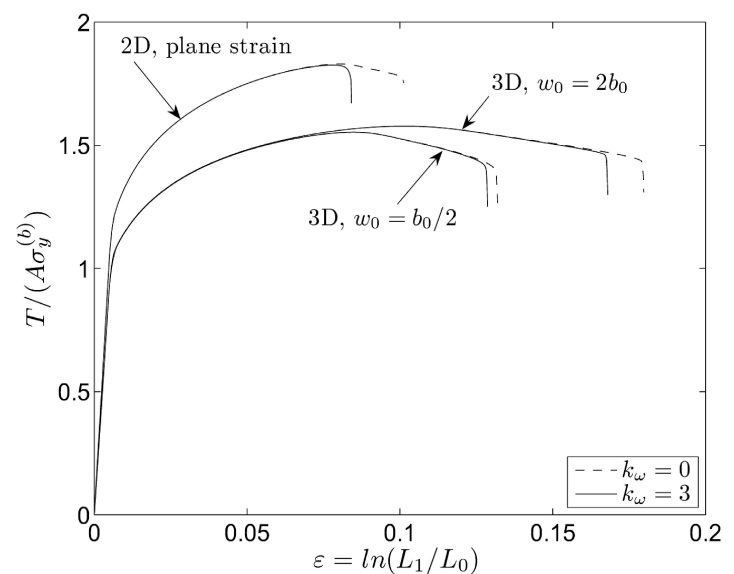

Fig. 5: Load vs. axial strain curves for friction stir welded specimens (from [94])

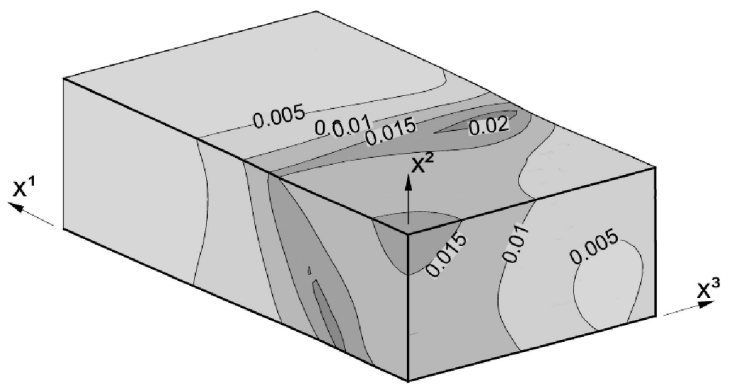

(a)

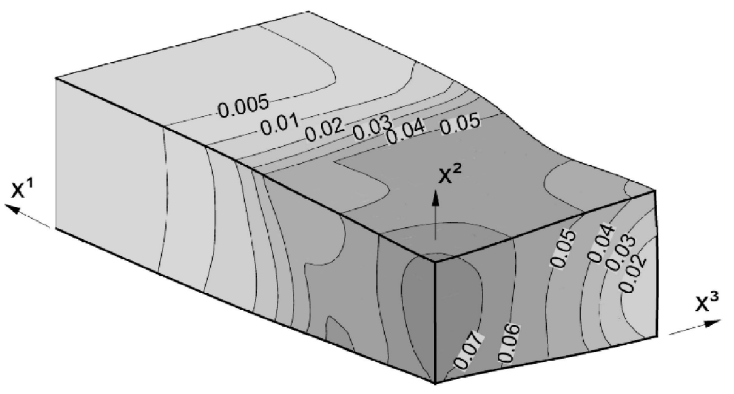

(b)

Fig. 6: Damage development, $f$, in a wide test specimen, $w_{0}=4 b_{0} ;\left(\right.$ a) at $\epsilon=0.1648 ;$ (b) at $\epsilon=0.2139\left(k_{\omega}=3\right)$ (from [94])

used in an experimental investigation to measure the distribution of the initial yield stress. Around the circular weld there is a weld nugget, approximately shaped as an ellipsoid, which has significantly higher yield stress than that in the base material.

In [96] the ductile fracture of shear-lab specimens has been analyzed by the GTN model as well as by the 
modified model from [44]. Fig. 7 shows the two types of failure modes that are predicted by the ductile fracture analyses, and also found in experiments. Results of a numerical solution obtained by the GTN model $\left(k_{\omega}=0\right)$ are shown in Fig. 8, presented as variations of field variables in the central cross-section along the specimen. As is seen from Fig. 7(a), this situation where plug failure is developing is highly nonlinear and three dimensional, not only in terms of large strains but also the weld nugget undergoes large rotations and the material beside the weld nugget deforms in ways very different from that in the nugget. As seen in Fig. 8 there is practically no straining and no void growth inside the weld nugget, where the yield stress is higher, and failure is growing in the softer material beside the nugget, which is going to develop into the shear failure that will separate the nugget from the rest of the specimen, as is characteristic for plug failure.

Fig. 9 shows curves of tensile force vs. end displacement as predicted by four different material models, where D is the GTN model, and A the modified model from [44]. Models B and C are versions of A, where the extra damage term is only activated in intervals of low stress triaxiality $T$, in the vicinity of $T=0$. The curve for model D corresponds to the computation also illustrated in Fig. 8. Since the stress triaxialities in Fig. 8(b) are rather low, this is in the range where the extra damage term introduced in [44] is expected to play a noticeable role, and therefore it is not surprising that material A predicts failure a great deal earlier than material D. It is noted that in [97] computations like those in Fig. 9 have also been carried out for the GLD model, thus accounting for the effect of void shape changes, and these computations have predicted later failure than that for model D in Fig. 9.

The computations illustrated in Figs. 8 and 9 are carried out for a specimen with weld radius $a=4 \mathrm{~mm}$, specimen width $2 w=25 \mathrm{~mm}$, and sheet thickness $t=$ $1.5 \mathrm{~mm}$. In [96] computations have also been carried out for a lower weld radius, $a=1.5 \mathrm{~mm}$, on the same shearlab specimen. Here the area of the weld is so small that the force needed to shear off the weld nugget is not sufficient to bend the plates in the shear-lab specimen near the weld and therefore the interfacial failure mode occurs as illustrated in Fig. 7(b). The material in the weld nugget undergoes essentially simple shear with the stress triaxiality $T=0$, and as expected model D (the GTN model) predicts no failure, as there is no void growth, while model A predicts failure at an end displacement and a tensile force much smaller than found in Fig. 9.

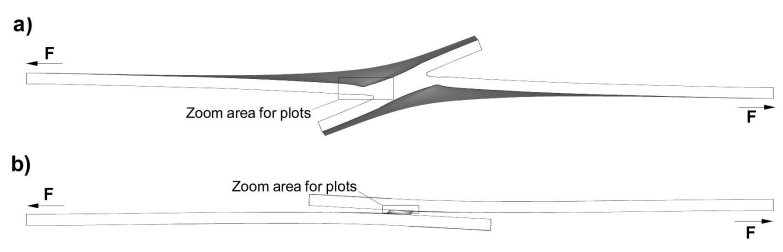

Fig. 7: Examples of modelled shear-lab specimens; (a) the plug failure mode; (b) the interfacial failure mode (from [96])

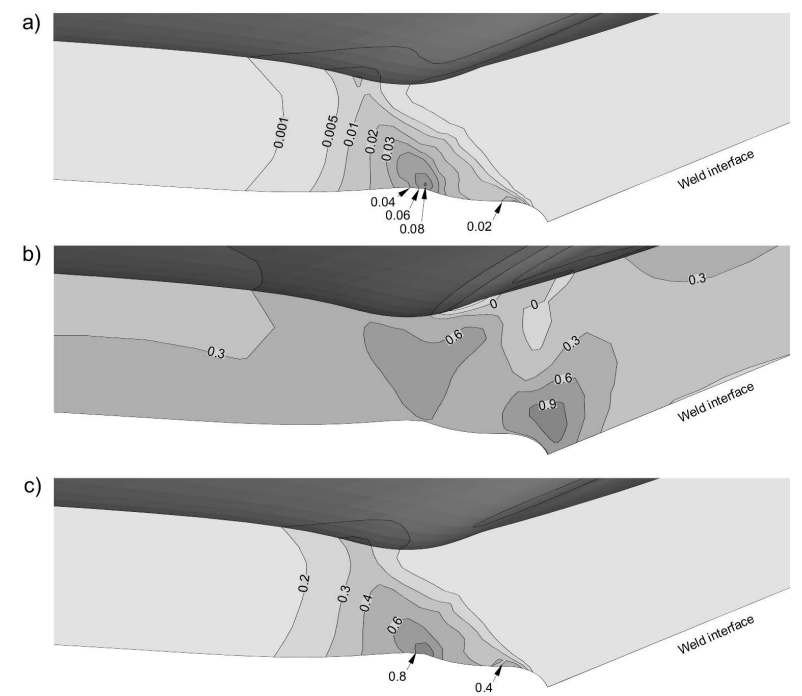

Fig. 8: Plug failure of single spot welded shear-lab specimen with weld radius $a=4 \mathrm{~mm}$, specimen width $2 w=25 \mathrm{~mm}$, and sheet thickness $t=1.5 \mathrm{~mm}-$ for model $\mathrm{D}\left(k_{\omega}=0\right)$; (a) void volume fraction $f$; (b) stress triaxiality $T$; (c) microscopic plastic strain $\epsilon_{M}^{p}$ (from [96])

7.2 Simulations of Dunand and Mohr's experiments on "butterfly" specimens - Jean-Baptiste

Dunand and Mohr [98,99] have recently developed a technique for experimental study of thin structures. The optimized "butterfly" specimens this technique is based on are basically plates, the central region of which is made thinner in order to concentrate the strain and control the location of the crack. These specimens are subjected to combined tension and shear; the loading may be proportional or non-proportional, and vary between the extremes of tension in transverse plane strain and pure shear. Details may be found in Dunand and Mohr [98, 99].

In the experiments considered hereafter, the ratio $F_{V} / F_{H}$ of the vertical (tensile) to horizontal (shear) forces is kept constant. Four values of the angle $\beta \equiv$ $\arctan \left(F_{V} / F_{H}\right)$ are considered, $\beta=90^{\circ}$ (tension in 


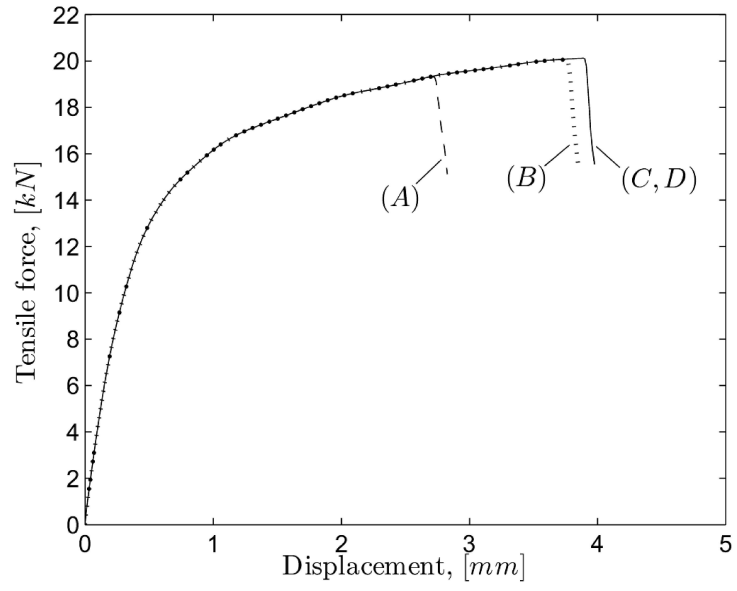

Fig. 9: Modelled tensile curves for single spot welded shear-lab specimen with weld radius $a=4 \mathrm{~mm}$, specimen width $2 w=25 \mathrm{~mm}$, and sheet thickness $t=$ $1.5 \mathrm{~mm}$ - in all cases ductile plug failure was predicted (from [96])

transverse plane strain), $63^{\circ}, 25^{\circ}$ and $0^{\circ}$ (pure shear). For each experiment, two load-displacement curves are recorded: vertical force versus vertical displacement and horizontal force versus horizontal displacement. Photographs of the fractured specimens are also taken. The material used is a high resistance TRIP780 steel. Again, details are provided in Dunand and Mohr [98,99].

The simulations are performed using the ABAQUS finite element programme. The 3D mesh consists of 71986 trilinear, selectively subintegrated (C3D8) elements and 82479 nodes. Controlled displacements are imposed on the lower and upper surfaces of the specimen. The model used is that of Madou and Leblond [48-51] accounting for void shape effects, well adapted to conditions of low triaxiality, see Section 3.3.2 above. Coalescence of voids is accounted for in the heuristic manner suggested by Tvergaard and Needleman's [18]. The main uncertainties in the simulations arise from the fact that void nucleation is disregarded; this phenomenon is known to be important in the type of steel considered although the relevant parameters are not known precisely.

Figure 10 shows the experimental (Exp) and computed (ML) load-displacement curves for various values of the angle $\beta$ defined above. (The case $\beta=0^{\circ}$ is disregarded in Fig. 10a because the vertical force and displacement are zero then, and the case $\beta=90^{\circ}$ is similarly disregarded in Fig. 10b). All experimental curves are acceptably reproduced. (The agreement could be improved by accounting for the plastic anisotropy of the material through use of Morin et al.'s [83] model, see Section 3.4.3). The most remarkable point here is the reproduction of the experimental final instability: the computed load-displacement curve becomes more or less suddenly vertical, and the elastoplastic iterations cease to converge. This occurs even for very low triaxialities $\left(\beta=0^{\circ}\right.$ or $\left.25^{\circ}\right)$, because of the damage induced by void shape changes. The prediction of this type of damage is a typical feature of Madou and Leblond's [48-51] model - simulations performed with the GTN model not accounting for void shape effects have been performed and checked to fail to reproduce, for low triaxialities, the final experimental instability.

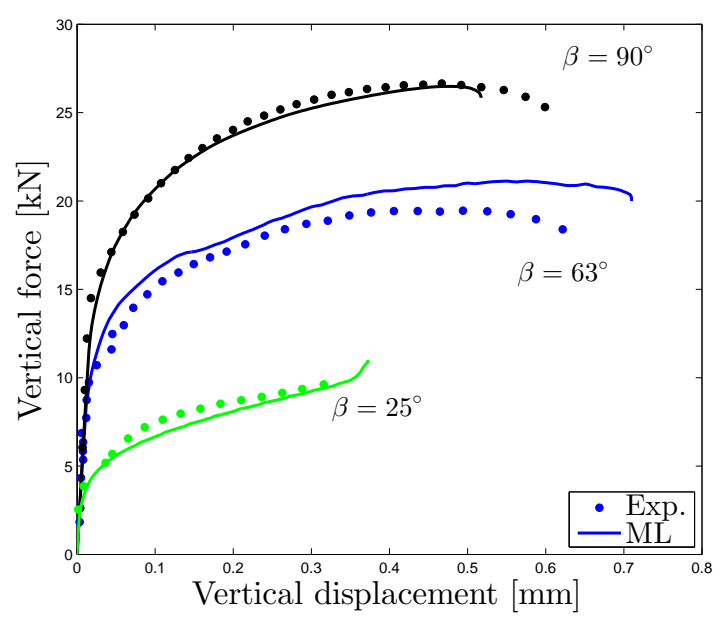

(a) Vertical force vs. vertical displacement

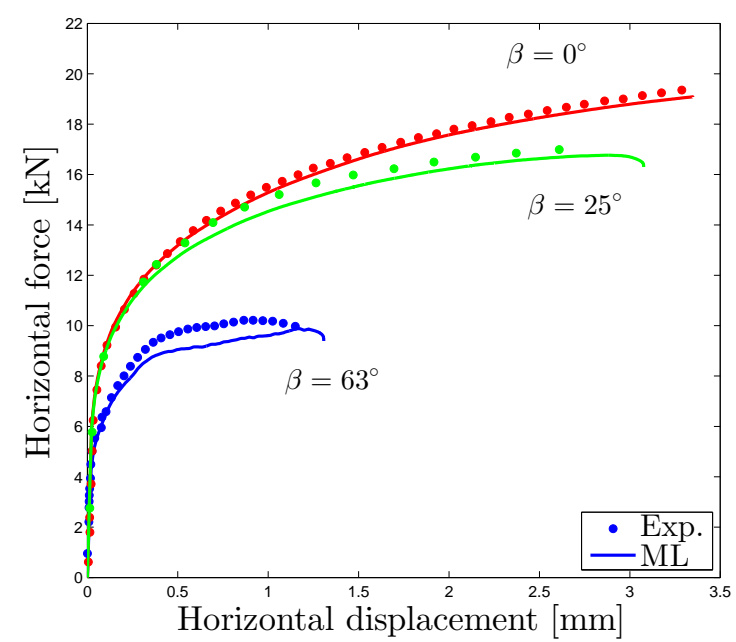

(b) Horizontal force vs. horizontal displacement

Fig. 10: Butterfly specimen - Vertical force vs. vertical displacement and horizontal force vs. horizontal displacement 
Figure 11 compares, in the case $\beta=25^{\circ}$, the photograph of the fractured specimen and the distribution of the maximum principal strain computed at the last available instant (just prior to the instability), on the deformed configuration (without any amplification of the displacements). The location of the yellow and red region where the computed maximum principal strain takes its highest values coincides quite well with that of the experimentally observed crack. More, the black circle of Fig. 11a, which marks the experimental point of initiation of the crack, lies precisely within the red zone of Fig. 11b where the strain is maximum.

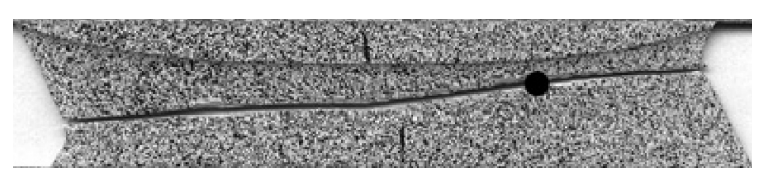

(a) Fractured specimen

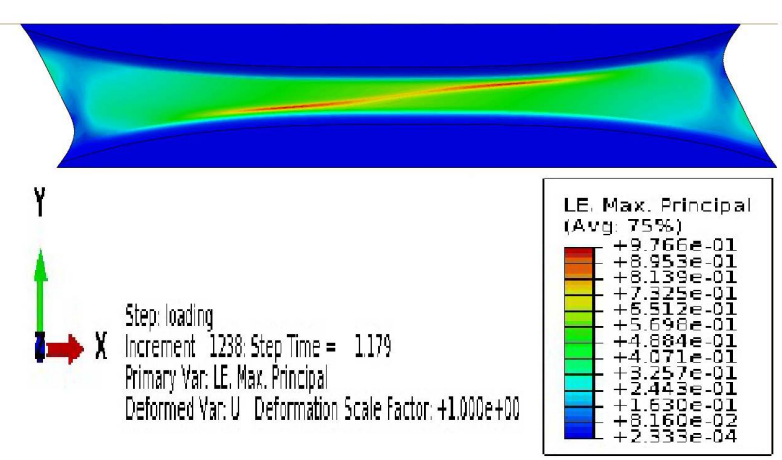

(b) Distribution of maximal principal strain

Fig. 11: Butterfly specimen - Photograph of the fractured specimen and computed distribution of the maximum principal strain

Numerical simulations of these experiments have also been successfully performed by Dunand and Mohr [98] using Gurson's [13] model with Nahshon and Hutchinson's [44] heuristic modification of the evolution equation of the porosity, see Section 3.2.2. The simulations just presented show that use of Madou and Leblond's [48-51] model accounting for void shape effects represents a viable alternative, more rigorous albeit more complex, way of reproducing Dunand and Mohr's [98, 99] experiments.
7.3 Two applications of Gologanu et al.'s second-gradient extension of Gurson's model Jean-Baptiste

The predictions of Gologanu et al.'s [22] second gradient extension of Gurson's [13] model, aimed at solving the problem of unlimited localization of strain and damage, see Section 3.5, will now be illustrated through two numerical examples due to Bergheau et al. [86]. All computations described in this section have been performed using the SYSTUS finite element programme developed by ESI-Group.

The first example pertains to the $2 \mathrm{D}$ axisymmetric simulation of a fracture test performed on a pre-notched and precracked TA30 specimen $(\mathrm{TA}=$ Tensile Axisymmetric, $30=$ diameter in $\mathrm{mm}$ ). The geometry and one of its discretizations are represented in Fig. 12. Advantage is taken of symmetry about the horizontal mid-plane to model only the upper half of the specimen, and the axis of rotational symmetry coincides with the left boundary of the mesh; note the triangular notch at the lower-right corner, from which the horizontal fatigue pre-crack (invisible in the figure) originates. Two meshes are in fact used, so as to permit a study of the mesh sensitivity of the results; they differ through the size of the square elements used in the region of the propagating crack, 0.3 $\mathrm{mm}$ and $0.1 \mathrm{~mm}$, the mesh represented in Fig. 12 corresponding to the former value. The specimen is made of A 508 Class 3 steel (used in nuclear components); the material parameters are provided in Bergheau et al. [86]. In particular, various values of Tvergaard and Needleman's [18] heuristic parameters of coalescence $f_{c}$ ("critical porosity" at the onset of coalescence) and $\delta$ ("accelerating factor") are used, and the value of the "microstructural distance" $b$ (see Section 3.5) is 0.55 $\mathrm{mm}$.

Figure 13 compares the experimental load-displacement curve (in dark blue) to various numerical ones:

- The yellow and green curves have been obtained with the same coalescence parameters, $f_{c}=0.08$ and $\delta=4$ but the two meshes having element sizes of 0.3 and $0.1 \mathrm{~mm}$ in the region of the crack, respectively. The curves are very close, showing that the influence of the discretization is minimal. Results obtained with Gurson's [13] standard first-gradient model would exhibit a much larger mesh sensitivity in the descending portion of the curve.

- The red curve corresponds to the best agreement obtained with the experimental results; it has been obtained with the mesh having an element size of $0.3 \mathrm{~mm}$ in the region of the crack and the parameters $f_{c}=0.04$ and $\delta=2$. The agreement with the experimental results is quite acceptable considering 


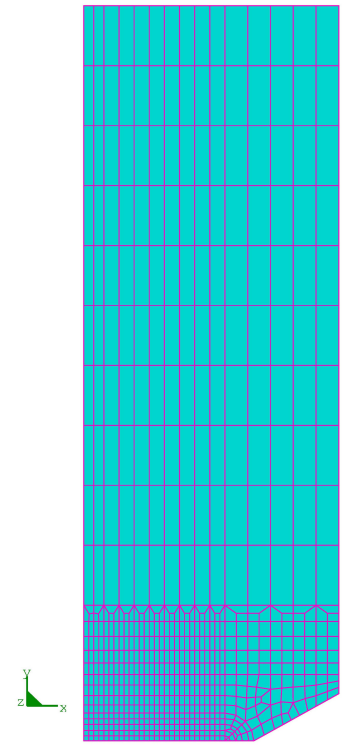

Fig. 12: Mesh of a TA30 specimen

the inherently imperfect reproducibility of experimental results. This result is remarkable in view of the qualitative compatibility of the value of $f_{c}$ used with the theoretical ones, of the order of a few percent, obtained by various authors through micromechanical finite element simulations of representative porous cells, see the pioneering work of Koplik and Needleman [28] and its many successors. Such a result could never be obtained with Gurson's model which was always observed to necessitate much smaller, and unrealistic values of $f_{c}$ to warrant satisfactory reproduction of experimental load-displacement curves of cracked specimens.

- The brown curve has been obtained with the same mesh but coalescence has been suppressed here by adopting a high value for $f_{c}$ (or equivalently a unity value for $\delta$ ). The large discrepancy with the experimental curve, especially in its descending portion, illustrates the necessity of accounting for coalescence to satisfactorily reproduce the test.

- Finally the light blue curve has been obtained with the same mesh and coalescence parameters as the red one, but using Gurson's [13] original model instead of Gologanu et al.'s [22] second gradient extension. The much-too-modest decrease of the load illustrates the incapacity of Gurson's model to reproduce experimental results for such high values of $f_{c}$, and the necessity of using much lower, unrealistic values.

The second example pertains to the $2 \mathrm{D}$ plane strain simulation of a fracture test performed on a CT12 specimen $(\mathrm{CT}=$ Compact Test, $12=$ thickness in $\mathrm{mm})$.

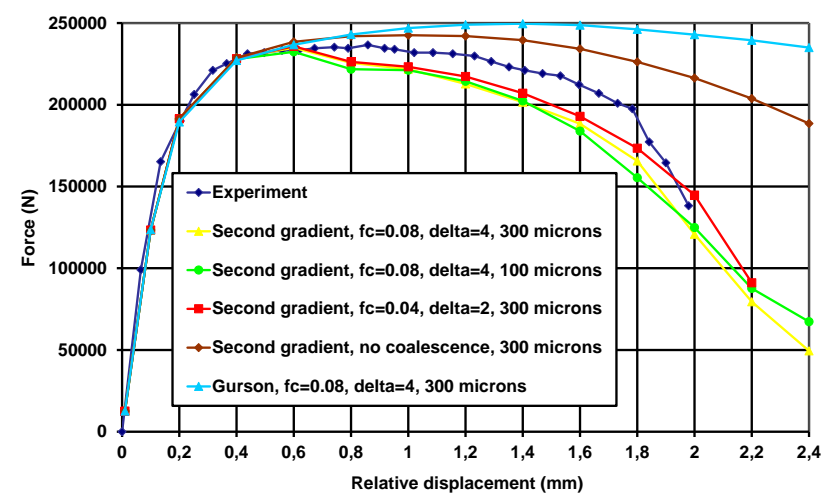

Fig. 13: Load-displacement curves of a TA30 specimen

The discretized geometry is shown in Fig. 14. (A single mesh is used this time). Advantage is taken of symmetry about the vertical mid-plane of the specimen to model only its right half. A vertical fatigue pre-crack (invisible in the figure) originates from the notch root. Square elements of size $0.3 \mathrm{~mm}$ are used in the region of the propagating crack. The specimen is made of SS $316 \mathrm{~L}$ stainless steel; the material parameters are provided in Bergheau et al. [86]. In particular, various values of Tvergaard and Needleman's [18] "critical porosity" at the onset of coalescence $f_{c}$ and "accelerating factor" $\delta$ are used again, and the value of the "microstructural distance" $b$ is $0.5 \mathrm{~mm}$.

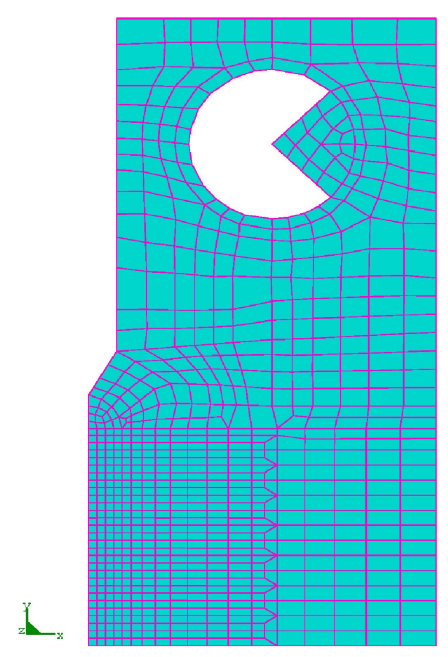

Fig. 14: Mesh of a CT12 specimen 
Figure 15 shows the experimental load-displacement curve (in dark blue) together with three numerical ones:

- The red curve has been obtained with Gologanu et al.'s [22] second gradient model, including coalescence with the values $f_{c}=0.05$ and $\delta=2$. The agreement with the experimental curve is excellent, again illustrating the model's capability to reproduce experimental results using relatively high values of $f_{c}$ compatible with the available theoretical estimates.

- The brown curve has been obtained with the same model but disregarding coalescence. Again, the large discrepancy with the experimental curve illustrates the necessity of accounting for coalescence to satisfactorily reproduce the test.

- The light blue curve has been obtained with the same coalescence parameters as the red one but using Gurson's [13] model. The large gap between this curve and the experimental one again illustrates the incapacity of Gurson's model to reproduce experimental results for high, realistic values of $f_{c}$.

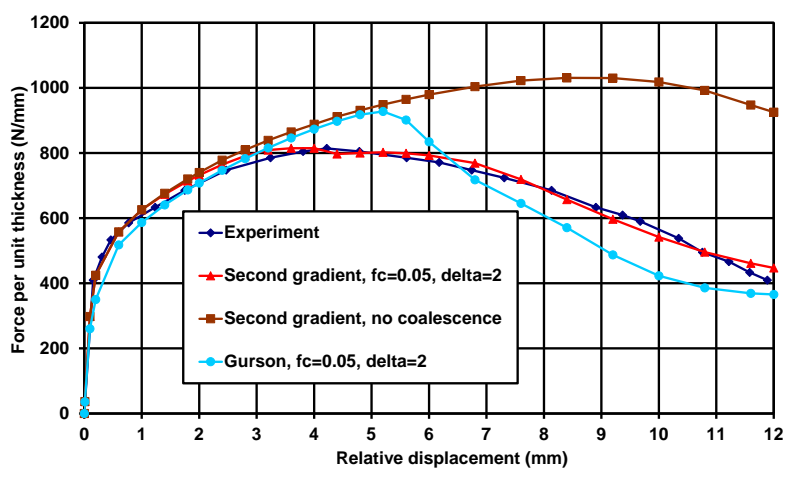

Fig. 15: Load-displacement curves of a CT12 specimen

Figure 15 shows the distribution of the opening stress (perpendicular to the crack plane) on the deformed configuration of the specimen (without any magnification of the displacements), at the last instant of the simulation corresponding to the red curve in Figure 15. (The undeformed mesh is shown in the background for reference). The important propagation of the crack and the large ensuing deformation of the specimen are quite conspicuous here. On the vertical plane of symmetry, the opening stress is zero in the region of the crack, positive just ahead of the crack tip and negative beyond, as expected since the total moment of external forces must be zero.

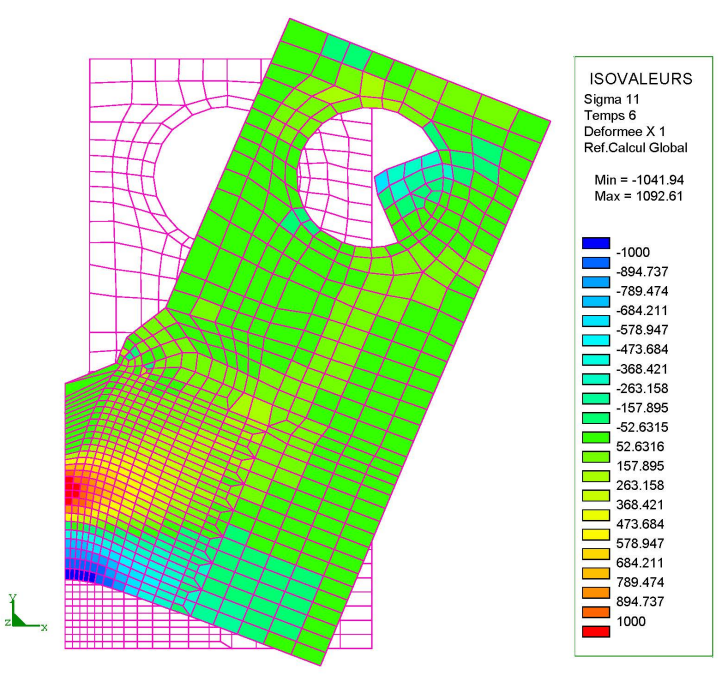

Fig. 16: Opening stress field in a CT12 specimen

Calculations have also been performed very recently using a mix of elements obeying von Mises's standard first-gradient model and Gologanu et al.'s [22] secondgradient extension of Gurson's [13] model, the idea being to reduce the CPU time by using the second-gradient model only in the region of the propagating crack where strain gradients are maximum. As explained in Section 3.5, Bergheau et al.'s [86] very recent numerical implementation of Gologanu et al.'s [22] second gradient model is especially fit to such a mix. Figure 17a shows three possible zones for the "second-gradient region", and Fig. 17b compares the corresponding loaddisplacement curves to that obtained using the secondgradient model in the whole structure, which serves as a reference. As can be seen, results virtually identical to the reference ones are obtained by confining secondgradient elements to "Zone 3" of Fig. 17a, with a CPUtime reduction of $24 \%$, and quite acceptable ones by confining second-gradient elements to "Zone 2", with a CPU-time reduction of $44 \%$. A more detailed inspection of results (not displayed here) shows that the same conclusions hold for local quantities such as stresses.

\section{References}

1. Tipper, C.F., 1949. The fracture of metals. Metallurgia, 39, 133-137. 


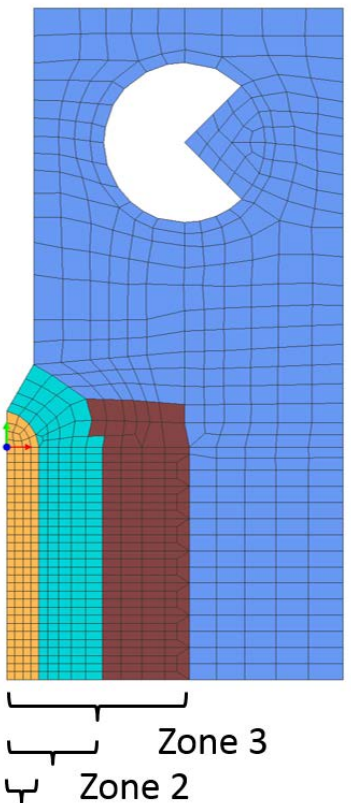

Zone 1

(a) Second gradient zones

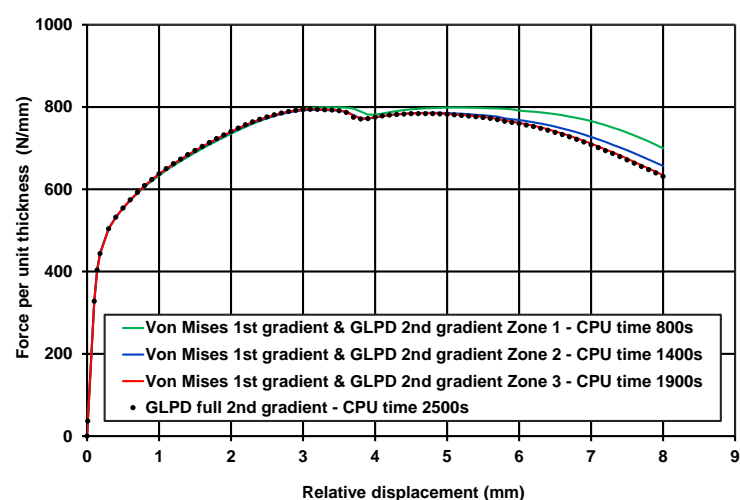

(b) Load-displacement curves

Fig. 17: CT specimen - Comparison of simulations using a second-gradient and a mix of first- and secondgradient models

2. Rogers, H.C., 1960. The tensile fracture of ductile metals. Trans. Metall. Soc. AIME, 218, 498-506.

3. Beachem, C.D., 1963. An electron fractographic study of the influence of plastic strain conditions upon ductile rupture processes in metals. Trans. ASM, 56, 318-326.

4. Puttick, K.E., 1959. Ductile fracture in metals. Phil. Mag., 4, 964-969.

5. Gurland, J. and Plateau, J., 1963. The mechanism of ductile rupture of metals containing inclusions. Trans. ASM, $56,442-454$.
6. Garrison Jr, W.M. and Moody, N.R., 1987. Ductile fracture. J. Phys. Chem. Solids 48, 1035-1074.

7. Tvergaard, V., 1990. Material failure by void growth to coalescence. Adv. Appl. Mech. 27, 83-151.

8. Benzerga, A. and Leblond, J.B., 2010. Ductile fracture by void growth to coalescence. Adv. Appl. Mech. 44, 169305.

9. McClintock, F.A., 1968. A criterion for ductile fracture by growth of holes. ASME J. Appl. Mech. 35, 363-371.

10. Rice, J.R. and Tracey, D.M., 1969. On the ductile enlargement of voids in triaxial stress fields. J. Mech. Phys. Solids 17, 201-217.

11. Needleman, A., 1972. Void growth in an elastic-plastic medium. ASME J. Appl. Mech. 41, 964-970.

12. Gurson, A.L., 1975. Plastic flow and fracture behavior of ductile materials incorporating void nucleation, growth and interaction. Ph.D. Thesis, Brown University.

13. Gurson, A.L., 1977. Continuum theory of ductile rupture by void nucleation and growth - I. Yield criteria and flow rules for porous ductile media. ASME J. Engng. Mater. Technol. 99, 2-15.

14. Bishop, R.F., Hill, R. and Mott, N.F., 1945. The theory of indentation and hardness tests. Proc. Phys. Soc. London 57, 147-159.

15. Chu, C.C. and Needleman, A., 1980. Void nucleation effects in biaxially stretched sheets. J. Engng. Mater. Technol. 102, 249-256.

16. Tvergaard, V., 1981. Influence of voids on shear band instabilities under plane strain conditions. Int. J. Fract. 17, 389-407.

17. Tvergaard, V., 1982. On localization in ductile materials containing spherical voids. Int. J. Fract. 18, 237-252.

18. Tvergaard, V. and Needleman, A., 1984. Analysis of the cup-cone fracture in a round tensile bar. Acta. Metall. 32, 157-169.

19. Shima, S. and Oyane, M., 1976. Plasticity theory for porous metals. Int. J. Mech. Sci. 18, 285-291.

20. Gologanu, M., Leblond, J.-B. and Devaux, J., 1993. Approximate models for ductile metals containing nonspherical voids - Case of axisymmetric prolate ellipsoidal cavities. J. Mech. Phys. Solids 41, 1723-1754.

21. Gologanu, M., Leblond, J.-B. and Devaux, J., 1994. Approximate models for ductile metals containing nonspherical voids - Case of axisymmetric oblate ellipsoidal cavities. J. Engng. Mater. Technol. 116, 290-297.

22. Gologanu, M., Leblond, J.-B., Devaux, J. and Perrin, G., 1997. Recent extensions of Gurson's model for porous ductile metals. In: Suquet, ed., Continuum Micromechanics, CISM Courses and Lectures 377, Springer, 61-130.

23. Ponte-Castaneda, P. and Zaidman, M., 1994. Constitutive models for porous materials with evolving microstructure. J. Mech. Phys. Solids 42, 1459-1492.

24. Benzerga, A.A. and Besson, J., 2001. Plastic potentials for anisotropic porous solids. Eur. J. Mech. A/Solids 20, 397-434.

25. Hill, R., 1948. A theory of the yielding and plastic flow of anisotropic metals. Proc. Roy. Soc. London A 193, 281297.

26. Keralavarma, S. and Benzerga, A., 2008. An approximate yield criterion for anisotropic porous media. ComptesRendus Mecanique 336, 685-692.

27. Keralavarma, S. and Benzerga, A., 2010. A constitutive model for plastically anisotropic solids with non-spherical voids. J. Mech. Phys. Solids 58, 874-901.

28. Koplik, J. and Needleman, A., 1988. Void growth and coalescence in porous plastic solids. Int. J. Solids Struct. $24,835-854$. 
29. Rice, J.R., 1976. The localization of plastic deformation. In: Proceedings of the International Congress on Theoretical and Applied Mechanics, North-Holland, Amsterdam, 207-220.

30. Needleman, A. and Rice, J.R., 1978. Limits to ductility set by plastic flow localization. In: Koistinen et al., eds., Mechanics of Sheet Metal Forming, Plenum Publishing, 237-267.

31. Cox, T.B. and Low, J.R., 1974. An investigation of the plastic fracture in AISI 4340 and 18 nickel-200 grade maraging steels. Metall. Trans. 5, 1457-1470.

32. Stone, R.H. and Psioda, J.A., 1975. Discussion of: Metallurgical factors affecting fracture toughness of aluminum alloys. Metall. Trans. 6A, 668-670.

33. Tvergaard, V., 1982. Ductile fracture by cavity nucleation between larger voids. J. Mech. Phys. Solids 30, 265-286.

34. Barsoum, I. and Faleskog, J., 2007a. Rupture mechanisms in combined tension and shear - Micromechanics. Int. J. Solids Struct. 44, 5481-5498.

35. Barsoum, I. and Faleskog, J., 2007b. Rupture mechanisms in combined tension and shear - Experiments. Int. J. Solids Struct. 44, 1768-1786.

36. Tvergaard, V., 2008. Shear deformation of voids with contact modeled by internal pressure. Int. J. Mech. Sci. $50,1459-1465$.

37. Tvergaard, V., 2009. Behaviour of voids in a shear field. Int. J. Fract. 158, 41-49.

38. Tvergaard, V., 2012. Effect of stress-state and spacing on voids in a shear-field. Int. J. Solids Struct. 49, 3047-3054.

39. Dahl, J., Nielsen, K.L. and Tvergaard, V., 2012. Effect of contact conditions on void coalescence at low stress triaxiality shearing. ASME J. Appl. Mech. 79, 021003.

40. Nielsen, K.L., Dahl, J. and Tvergaard, V., 2012. Collapse and coalescence of spherical voids subject to intense shearing: Studied in full 3D. Int. J. Fract. 177, 97-108.

41. Bao, Y. and Wierzbicki, T., 2004. On fracture locus in the equivalent strain and stress triaxiality space. Int. J. Mech. Sci. 46, 81-98.

42. Barsoum, I. and Faleskog, J., 2011. Micromechanical analysis on the influence of the Lode parameter on void growth and coalescence. Int. J. Solids Struct. 48, 925-938.

43. Xue, Z., Faleskog, J. and Hutchinson, J.W., 2013. Tension-torsion fracture experiments - Part II: Simulations with the extended Gurson model and a ductile fracture criterion based on plastic strain. Int. J. Solids Struct. $50,4258-4269$.

44. Nahshon, K., and Hutchinson, J.W., 2008. Modification of the Gurson model for shear failure. Eur. J. Mech./A Solids 27, 1-17.

45. Tvergaard, V. and Nielsen, K.L., 2010. Relations between a micro-mechanical model and a damage model for ductile failure in shear. J. Mech. Phys. Solids 58, 1243-1252.

46. Monchiet, V., Gruescu, C., Charkaluk, E. and Kondo, D., 2006. Approximate yield criteria for anisotropic metals with prolate or oblate voids. Comptes-Rendus Mecanique 334, 431-439.

47. Monchiet, V., Cazacu, O., Charkaluk, E. and Kondo, D., 2008. Macroscopic yield criteria for plastic anisotropic materials containing spheroidal voids. Int. J. Plasticity $24,1158-1189$

48. Madou, K. and Leblond, J.-B., 2012a. A Gurson-type criterion for porous ductile solids containing arbitrary ellipsoidal voids - I: Limit-analysis of some representative cell. J. Mech. Phys. Solids 60, 1020-1036.

49. Madou, K. and Leblond, J.-B., 2012b. A Gurson-type criterion for porous ductile solids containing arbitrary ellipsoidal voids - II: Determination of yield criterion parameters. J. Mech. Phys. Solids 60, 1037-1058.
50. Madou, K. and Leblond, J.-B., 2013. Numerical studies of porous ductile materials containing arbitrary ellipsoidal voids - I: Yield surfaces of representative cells. Eur. J. Mech. A/Solids 42, 480-489.

51. Madou, K., Leblond, J.-B. and Morin, L., 2013. Numerical studies of porous ductile materials containing arbitrary ellipsoidal voids - II: Evolution of the length and orientation of the void axes. Eur. J. Mech. A/Solids 42, 490-507.

52. Mandel., J., 1964. Contribution théorique à l'étude de l'écrouissage et des lois de l'écoulement plastique. In: Proceedings of the 11th International Congress on Applied Mechanics, Munich, pp. 502-509.

53. Hill, R., 1967. The essential structure of constitutive laws for metal composites and polycrystals. J. Mech. Phys. Solids 15, 79-95.

54. Ponte-Castaneda, P., 1991. The effective mechanical properties of nonlinear isotropic composites. J. Mech. Phys. Solids 39, 45-71.

55. Willis, J., 1991. On methods for bounding the overall properties of nonlinear composites. J. Mech. Phys. Solids 39, $73-86$.

56. Michel, J.-C. and Suquet, P., 1992. The constitutive law of nonlinear viscous and porous materials. J. Mech. Phys. Solids 40, 783-812.

57. Danas, K. and Ponte-Castaneda, P., 2009a. A finitestrain model for anisotropic viscoplastic porous media: I - Theory. Eur. J. Mech. A/Solids 28, 387-401.

58. Danas, K. and Ponte-Castaneda, P., 2009b. A finitestrain model for anisotropic viscoplastic porous media: II - Applications. Eur. J. Mech. A/Solids 28, 402-416.

59. Ponte-Castaneda, P., 2002. Second-order homogenization estimates for nonlinear composites incorporating field fluctuations. I. Theory. J. Mech. Phys. Solids 50, 737757.

60. Agoras, M. and Ponte-Castaneda, P., 2013. Iterated linear comparison bounds for viscoplastic porous materials with "ellipsoidal" microstructures. J. Mech. Phys. Solids 61, 701-725.

61. Agoras, M. and Ponte-Castaneda, P., 2014. Anisotropic finite-strain models for porous viscoplastic materials with microstructure evolution. Int. J. Solids Structures 51, 981-1002.

62. Ponte-Castaneda, P., 2012. Bounds for nonlinear composites via iterated homogenization. J. Mech. Phys. Solids 60, 1583-1604.

63. Leblond, J.-B. and Morin, L., 2014. Gurson's criterion and its derivation revisited. ASME J. Appl. Mech. 81, 051012 .

64. Leblond, J.-B., Perrin, G. and Devaux, J., 1995. An improved Gurson-type model for hardenable ductile metals. Eur. J. Mech. A/Solids 14, 499-527.

65. Mear, M. and Hutchinson, J., 1985. Influence of yield surface curvature on flow localization in dilatant plasticity. Mech. Mater. 4, 395-407.

66. Tvergaard, V., 1987. Effect of yield surface curvature and void nucleation on plastic flow localization. J. Mech. Phys. Solids 35, 43-60.

67. Perrin, G. and Leblond, J.-B., 1990. Analytical study of a hollow sphere made of porous plastic material and subjected to hydrostatic tension - Application to some problems in ductile fracture of metals. Int. J. Plasticity 6, 677-699.

68. Huang, Y., 1991. Accurate dilatation rates for spherical voids in triaxial stress fields. ASME J. Appl. Mech. 58, 1084-1086. 
69. Gologanu, M., 1997. Etude de quelquesproblèmes de rupture ductile des métaux. Thèse de l'Université Pierre et Marie Curie (Paris VI).

70. Monchiet, V., Charkaluk, E. and Kondo, D., 2011. A micromechanics-based modification of the Gurson criterion by using Eshelby-like velocity fields. Eur. J. Mech. A/Solids 30, 940-949.

71. Cazacu, O., Revil-Baudard, B., Chandola, N. and Kondo, D., 2014. New analytical criterion for porous solids with Tresca matrix under axisymmetric loadings. Int. J. Solids Structures 51, 861-874.

72. Garajeu, M., Michel, J.-C. and Suquet, P., 2000. A micromechanical approach of damage in viscoplastic materials by evolution in size, shape and distribution of voids. Comput. Meth. Appl. Mech. Engng. 183, 223-246.

73. Lee, B. and Mear, M., 1992. Axisymmetric deformation of power-law solids containing a dilute concentration of aligned spheroidal voids. J. Mech. Phys. Solids 40, 18051836.

74. Budiansky, B., Hutchinson, J. and Slutsky, S., 1982. Void growth and collapse in viscous solids. In: Mechanics of Solids, the Rodney Hill Anniversary Volume, H.G. Hopkins and M.J. Sewell, eds., Pergamon Press, Oxford, pp. 13-45.

75. Scheyvaerts, F., Onck, P., Tekoglu, C. and Pardoen, T., 2011. The growth and coalescence of ellipsoidal voids in plane strain under combined shear and tension. J. Mech. Phys. Solids 59, 373-397.

76. Leblond, J.B. and Gologanu, M., 2008. External estimate of the yield surface of an arbitrary ellipsoid containing a confocal void. Comptes-Rendus Mécanique 336, 813-819.

77. Kailasam, M. and Ponte-Castaneda, P., 1998. A general constitutive theory for linear and nonlinear particulate media with microstructure evolution. J. Mech. Phys. Solids 46, 427-465.

78. Morin, Léo, 2015. Influence des effets de forme et de taille des cavités, et de l'anisotropie plastique sur la rupture ductile. Ph.D. thesis, Université Pierre et Marie Curie (Paris 6).

79. Willis, J., 1977. Bounds and self-consistent estimates for the overall moduli of anisotropic composites. J. Mech. Phys. Solids 25, 185-202.

80. Idiart, M., 2007. Nonlinear sequential laminates reproducing hollow sphere assemblages. Comptes-Rendus Mécanique 335, 363-368.

81. Idiart, M., 2008. Modeling the macroscopic behavior of two-phase nonlinear composites by infinite-rank laminates. J. Mech. Phys. Solids 56, 2599-2617.

82. Morin, L., Madou, K., Leblond, J.B. and Kondo, D., 2014. A new technique for finite element limit-analysis of Hill materials, with an application to the assessment of criteria for anisotropic plastic porous solids. Int. J. Engng. Sci. 74, 65-79.

83. Morin, L., K., Leblond, J.B. and Kondo, D., 2015. A Gurson-type criterion for plastically anisotropic solids containing arbitrary ellipsoidal voids. To be published in Int. J. Solids Structures.

84. Leblond, J.B., Perrin, G. and Devaux J., 1994. Bifurcation effects in ductile metals with nonlocal damage. ASME J. Appl. Mech. 61, 236-242.

85. Enakoutsa, K. and Leblond, J.B., 2009. Numerical implementation and assessment of the GLPD micromorphic model of ductile rupture. Eur. J. Mech. A/Solids 28, 445460.

86. Bergheau, J.M., Leblond, J.B. and Perrin, G., 2014. A new numerical implementation of a second-gradient model for plastic porous solids, with an application to the simulation of ductile rupture tests. Comput. Meth. Appl. Mech. Engng. 268, 105-125.

87. Ritchie, R.O., 1978. On the relationship between fracture toughness and Charpy V-Notch energy in ultrahigh strength steels. In: Rosenfield A.R., Gegel H.L., Hasson D.F., editors. What does the Charpy test really tell us? American Society for Metals, pp. 54-73.

88. Francois, D. and Pineau, A., editors, 2002. From Charpy to present impact testing. Elsevier, Oxford.

89. Tvergaard, V. and Needleman, A., 1986. Effect of material rate sensitivity on failure modes in the Charpy Vnotch test. J. Mech. Phys. Solids 34, 213-241.

90. Tvergaard, V. and Needleman, A., 1988. An analysis of temperature and rate dependence of Charpy V-notch energies for a high nitrogen steel. Int. J. Fract. 37, 197-215.

91. Tvergaard, V. and Needleman, A., 2000. Analysis of the Charpy V-notch test for welds. Engng. Fract. Mech. 65, 627-643.

92. Tvergaard, V. and Needleman, A., 2004. 3D analyses of the effect of weld orientation in Charpy specimens. Engng. Fract. Mech. 71, 2179-2195.

93. Nielsen, K.L., 2008a. Ductile damage development in friction stir welded aluminum (AA2024) joints. Engng. Fract. Mech. 75, 2795-2811.

94. Nielsen, K.L., Tvergaard, V., 2009. Effect of a shear modified Gurson model on damage development in a FSW tensile specimen. Int. J. Solids Struct. 46, 587-601.

95. Nielsen, K.L., 2008b. 3D modelling of plug failure in resistance spot welded shear-lab specimens (DP600-steel). Int. J. Fract. 153, 125-139.

96. Nielsen, K.L., Tvergaard, V., 2010. Ductile shear failure or plug failure of spot welds modelled by modified Gurson model. Engng. Fract. Mech. 77, 1031-1047.

97. Nielsen, K.L., 2010. Predicting failure response of spot welded joints using recent extensions to the Gurson model. Comput. Mater. Sci. 48, 71-82.

98. Dunand, M. and Mohr, D., 2011. On the predictive capabilities of the shear modified Gurson and the modified Mohr-Coulomb fracture models over a wide range of stress triaxialities and Lode angles. J. Mech. Phys. Solids 59, 1374-1394.

99. Dunand, M. and Mohr, D., 2011. Optimized butterfly specimen for the fracture testing of sheet materials under combined normal and shear loading. Engng. Fract. Mech. 78, 2919-2934. 